Authors are encouraged to submit new papers to INFORMS journals by means of a style file template, which includes the journal title. However, use of a template does not certify that the paper has been accepted for publication in the named journal. INFORMS journal templates are for the exclusive purpose of submitting to an INFORMS journal and should not be used to distribute the papers in print or online or to submit the papers to another publication.

\title{
On a piecewise-linear approximation for network revenue management
}

\author{
Sumit Kunnumkal \\ Indian School of Business, Hyderabad, 500032, India, sumit_kunnumkal@isb.edu, \\ Kalyan Talluri \\ Imperial College Business School, London SW7 2AZ, UK, kalyan.talluri@imperial.ac.uk,
}

\begin{abstract}
The network revenue management (RM) problem arises in airline, hotel, media, and other industries where the sale products use multiple resources. It can be formulated as a stochastic dynamic program, but the dynamic program is computationally intractable because of an exponentially large state space, and a number of heuristics have been proposed to approximate its value function. In this paper we show that the piecewise-linear approximation to the network RM dynamic program is tractable; specifically we show that the separation problem of the approximation can be solved as a relatively compact linear program. Moreover, the resulting compact formulation of the approximate dynamic program turns out to be exactly equivalent to the Lagrangian relaxation of the dynamic program, an earlier heuristic method proposed for the same problem. We perform a numerical comparison of solving the problem by generating separating cuts or as our compact linear program. We discuss extensions to versions of the network RM problem with overbooking as well as the difficulties of extending it to the choice model of network revenue RM.
\end{abstract}

Key words: network revenue management, linear programming, approximate dynamic programming,

Lagrangian relaxation methods.

MSC2000 subject classification:

History:

Revenue management is the control of the sale of a limited quantity of a resource (hotel rooms for a night, airline seats, advertising slots etc.) to a heterogenous population with different valuations for a unit of the resource. The resource is perishable, and for simplicity sake, we assume that it perishes at a fixed point of time in the future. Sale is online, so the firm has to decide what products to offer at a given price for each product (so as not to sell too much at too low a price early and run out of capacity), or, reject too many low-valuation customers (and end up with excess unsold inventory).

In industries such as hotels, airlines and media, the products consume bundles of different resources (multi-night stays, multi-leg itineraries) and the decision to accept or reject a particular product at a certain price depends on the future demands and revenues for all the resources used by the product and, indirectly, on all the resources in the network. Network revenue management (network RM) involves making the acceptance decisions based on the product demands as well as the resource capacities for the entire network. Chapter 3 of Talluri and van Ryzin [18] contains all the necessary background on network RM.

The network RM problem can be formulated as a stochastic dynamic program, but computing the value function becomes intractable due to the high dimensionality of the state space. As a result, researchers have focused on developing approximation methods. In this paper we show that the 
piecewise-linear approximation to the network RM dynamic program is tractable; specifically we show that the separation problem can be solved as a linear program whose size is polynomial in $\max _{i}\left(r_{i}^{1}\right)$ where $r_{i}^{1}$ is capacity of resource $i$ in the network ${ }^{1}$. Moreover, the resulting compact formulation of the approximate dynamic program turns out to be exactly equivalent to the Lagrangian relaxation, an earlier heuristic method proposed for the same problem. We perform a numerical comparison of solving the problem by generating separating cuts or as our compact linear program. We discuss extensions to versions of the network RM problem with overbooking as well as the difficulties of extending it to the choice model of network revenue RM.

As a by-product, we derive some auxiliary results of independent interest: (i) we give a polynomialtime separation procedure for the piecewise-linear approximation linear program, (ii) we show that the optimal solution of the piecewise-linear approximation satisfies monotonicity conditions similar to that of a single-resource dynamic program, and (iii) sketch an extension to a model of network RM with overbooking.

The rest of the paper is organized as follows. In $\S 1$ we give a brief survey of the relevant literature. In $\S 2$ we first formulate the network RM problem as a dynamic program. We then describe the approximate dynamic programming approach with piecewise-linear basis functions and the previously proposed Lagrangian relaxation approach. In $\S 3$ we give the main body of proofs showing that the linear programming formulation of the piecewise-linear approximation is polynomial-time separable. In $\S 4$ we discuss a choice-model based network RM problem where we show the importance of formulation and customization of the number of Lagrange multipliers to the problem at hand. In $\S 5$ we give a small set of numerical results comparing the solution values and running times solving the piecewise-linear approximation by on-the-fly separation or as a compact linear program. In the appendix we give the proofs and also a sketch an extension to an overbooking model of network RM.

1. Relevant literature Approximate dynamic programming is the name given for methods that replace the value function of a (difficult) dynamic program (DP) with basis functions and solve the simplified problem as an approximation. In this stream of literature, the linear programming approach consists of formulating the dynamic program as a linear program with state-dependent variables representing the value functions and then replacing them by particular functional forms to find the best approximation within that class of functions. In the network RM context, this approach was first investigated by Adelman [1] who uses affine functions. A natural extension is to use piecewise-linear functions instead of affine as they are very flexible and indeed, for the single-resource dynamic program, optimal.

Another stream of literature revolves around the Lagrangian relaxation approach to dynamic programming. Here the idea is to relax certain constraints in the dynamic program by associating Lagrange multipliers with them so that the problem decomposes into simpler problems. For network RM, Topaloglu [20] and Kunnumkal and Topaloglu [11] take this approach. Topaloglu [20] uses Lagrange multipliers to relax the constraints that link the product acceptance decisions across the resources, while Kunnumkal and Topaloglu [11] relax the resource capacity constraints using Lagrange multipliers. Computational results from Topaloglu [20] indicate that Lagrangian relaxation with product-specific Lagrange multipliers gives consistent and clearly superior revenues compared to the other methods, including the affine approximation of Adelman [1]; the piecewise-linear approximation was not included, perhaps because it was not known how to solve it exactly. In a recent paper, Tong and Topaloglu [19] establish the equivalence between the affine approximation of Adelman [1] and the Lagrangian relaxation of Kunnumkal and Topaloglu [11]. A by-product of the results of this paper is that the product and

\footnotetext{
${ }^{1}$ So technically our algorithms are pseudo-polynomial as the size of the input is $\left.\log \left(\max _{i}\left(r_{i}^{1}\right)\right)\right)$. However in applications, $r_{i}^{1}$ is usually a small number so this is a minor technicality. Henceforth we simply say polynomial-time rather than polynomial in $\max _{i}\left(r_{i}^{1}\right)$.
} 
time-specific Lagrangian relaxation of Topaloglu [20] and the piecewise-linear approximation represent the same linear program.

Network RM incorporating more realistic models of customer behavior, as customers choosing from an offer set, have recently become popular after the single-resource model proposed in Talluri and van Ryzin [17]; see Gallego et al. [6], Liu and van Ryzin [13], Kunnumkal and Topaloglu [12], Meissner and Strauss [14], Bodea et al. [3], Bront et al. [4], Méndez-Díaz et al. [15] for subsequent extensions to network RM. In contrast to the independent demands setting described earlier, customers do not come in with the intention of purchasing a fixed product; rather their purchasing decision is influenced by the set of products that are made available for sale. This problem appears to be an order of magnitude more difficult to approximate than the independent-class network RM problem. Kunnumkal and Talluri [9] show that even the affine approximation of the dynamic program under the simplest possible choice model, a single-segment multinomial-logit model, is NP-complete.

2. Network revenue management We describe our notation and formulate the network RM problem as a dynamic program.

2.1. Notation and problem description A set of products on sale use a set of resources represented by edges of a network during $\tau$ time periods (booking horizon), with time being indexed from 1 to $\tau$. We assume that each product uses at most one unit of each resource.

The underlying network has $m$ resources (usually indexed by $i$ ) and $n$ products (usually indexed by $j$ ), and we refer to the set of all resources as $\mathcal{I}$ and the set of all products as $\mathcal{J}$. A product $j$ uses a subset of resources $\mathcal{I}_{j} \subseteq \mathcal{I}$, and its sale brings in revenue $f_{j}$. A resource $i$ is used by a subset $\mathcal{J}_{i} \subseteq \mathcal{J}$ of products. A resource $i$ is said to be in product $j\left(i \in \mathcal{I}_{j}\right)$ if $j$ uses resource $i$, and conversely we write $j \in \mathcal{J}_{i}$.

We use $\mathbb{1}_{[\cdot]}$ as the indicator function, 1 if true and 0 if false. The resources used by product $j$ are represented by the 0-1 incidence vector $\mathbb{1}_{\left[\mathcal{I}_{j}\right]}$, which has a 1 in the $i$ th position if $i \in \mathcal{I}_{j}$ and a 0 otherwise.

Booking requests for products come in over time and we let $p_{j, t}$ denote the probability that we get a request for product $j$ at time period $t$. This is the so-called independent demands model in the revenue management literature. We make the standard assumption that the time periods are small enough so that we get a request for at most one product in each time period. Throughout we assume that $p_{j, t}>0$ for all $j$. Note that this is without loss of generality because if $p_{j, t}=0$ for some product $j$, then we can simply discard that product and optimize over a smaller number of products. We also assume that $\sum_{j} p_{j, t}=1$ for all time periods $t$. This is also without loss of generality because we can add a dummy product with negligible revenue on each resource. We let $f_{j}$ denote the revenue associated with product $j$.

Given a request for product $j$, the firm has to decide online whether to accept or reject the request. An accepted request generates revenue and consumes capacity on the resources used by the product; a rejected request does not generate any revenue and simply leaves the system.

Throughout, we use boldface for vectors. We represent capacity vectors by $\boldsymbol{r}$. We use superscripts on vectors to index the vectors (for example, the resource capacity vector associated with time period $t$ would be $\boldsymbol{r}^{t}$ ) and subscripts to indicate components (for example, the capacity on resource $i$ in time period $t$ would be $r_{i}^{t}$ ).

We let $\boldsymbol{r}^{1}=\left[r_{i}^{1}\right]$ represent the initial capacity on the resources and $\boldsymbol{r}^{t}=\left[r_{i}^{t}\right]$ denote the remaining capacity on resource $i$ at time period $t$. The remaining capacity $r_{i}^{t}$ takes values in the set $\mathcal{R}_{i}=\left\{0, \ldots, r_{i}^{1}\right\}$ and $\mathcal{R}=\prod_{i} \mathcal{R}_{i}$ represents the state space. 
We represent control vectors by $\boldsymbol{u}$ and $\boldsymbol{u}^{t}=\left[u_{j}^{t}\right]$ represents a vector of controls at the network level at time $t$. We let $u_{j}^{t} \in\{0,1\}$ indicate the acceptance decision-1 if we accept product $j$ at time $t$ and 0 otherwise.

2.2. Dynamic Program The network RM problem can be formulated as a DP. Let

$$
\mathcal{U}(\boldsymbol{r})=\left\{\boldsymbol{u} \in\{0,1\}^{n} \mid u_{j} \leq r_{i} \forall j, i \in \mathcal{I}_{j}\right\},
$$

be the set of acceptable products when the state is $\boldsymbol{r}$. The value functions $V_{t}(\cdot)$ can be obtained through the optimality equations

$$
V_{t}(\boldsymbol{r})=\max _{\boldsymbol{u} \in \mathcal{U}(\boldsymbol{r})} \sum_{j} p_{j, t} u_{j}\left[f_{j}+V_{t+1}\left(\boldsymbol{r}-\sum_{i \in \mathcal{I}_{j}} \boldsymbol{e}^{i}\right)-V_{t+1}(\boldsymbol{r})\right]+V_{t+1}(\boldsymbol{r}),
$$

where $\boldsymbol{e}^{i}$ is a vector with a 1 in the $i$ th position and 0 elsewhere, and the boundary condition is $V_{\tau+1}(\cdot)=0$. Noting that $\boldsymbol{r}^{1}$ represents the initial capacity on the resources, $V_{1}\left(\boldsymbol{r}^{1}\right)$ gives the optimal expected total revenue over the booking horizon.

The value functions can, alternatively, be obtained by solving the linear program with an exponential number of decision variables $\left\{V_{t}(\boldsymbol{r}) \mid \forall t, \boldsymbol{r} \in \mathcal{R}\right\}$ and an exponential number of constraints:

$$
\begin{array}{rlr}
\min _{V} & V_{1}\left(\boldsymbol{r}^{1}\right) & \\
\text { S.t } & & \\
& V_{t}(\boldsymbol{r}) \geq \sum_{j} p_{j, t} u_{j}\left[f_{j}+V_{t+1}\left(\boldsymbol{r}-\sum_{i \in \mathcal{I}_{j}} \boldsymbol{e}^{i}\right)-V_{t+1}(\boldsymbol{r})\right]+V_{t+1}(\boldsymbol{r}) \\
& V_{\tau+1}(\cdot)=0 . & \forall t, \boldsymbol{r} \in \mathcal{R}, \boldsymbol{u} \in \mathcal{U}(\boldsymbol{r})
\end{array}
$$

Both the recursive equations $(1)$ as well as the linear program $\left(D P_{L P}\right)$ are intractable. In the following sections, we describe two approximation methods.

2.3. Piecewise-linear approximation for Network RM We approximate the value functions ${ }^{2}$ of (1) by

$$
V_{t}(\boldsymbol{r}) \approx \sum_{i} v_{i, t}\left(r_{i}\right), \forall \boldsymbol{r} \in \mathcal{R}
$$

Substituting this approximation into $\left(D P_{L P}\right)$, we obtain the linear program

$$
\begin{aligned}
& V^{P L}=\min _{v} \sum_{i} v_{i, 1}\left(r_{i}^{1}\right) \\
& \begin{aligned}
\sum_{i} v_{i, t}\left(r_{i}\right) \geq \sum_{j} p_{j, t} u_{j}\left[f_{j}+\sum_{i \in \mathcal{I}_{j}}\left\{v_{i, t+1}\left(r_{i}-1\right)-v_{i, t+1}\left(r_{i}\right)\right\}\right] \\
\quad+\sum_{i} v_{i, t+1}\left(r_{i}\right) \quad \forall t, \boldsymbol{r} \in \mathcal{R}, \boldsymbol{u} \in \mathcal{U}(\boldsymbol{r}) \\
v_{i, \tau+1}(\cdot)=0, v_{i, t}(-1)=-\infty, \forall t, i,
\end{aligned}
\end{aligned}
$$

where the decision variables are $\left\{v_{i, t}\left(r_{i}\right) \mid \forall t, i, r_{i} \in \mathcal{R}_{i}\right\}$. The number of decision variables in $(P L)$ is $\sum_{i} r_{i}^{1} \tau$ which is manageable. However, since $(P L)$ has an exponential number of constraints of type $(2)$, we need to use a separation algorithm to generate constraints on the fly to solve $(P L)$ (Grötschel et al. [7]). In $\S 3$, we show that the separation can be carried out efficiently for $(P L)$.

2 Adelman [1] uses the affine relaxation $V_{t}(\boldsymbol{r}) \approx \theta_{t}+\sum_{i} r_{i} v_{i, t}$ but we do not need the offset term $\theta_{t}$ for piecewise-linear approximations as we can use the transformation $\bar{v}_{i, t}\left(r_{i}\right)=\theta_{t} / m+v_{i, t}\left(r_{i}\right)$; see also Adelman and Mersereau [2]. 
A natural question is to understand the structural properties of an optimal solution to $(P L)$. This is useful since imposing structure can often significantly speed up the solution time. Lemma 1 below shows that an optimal solution to $(P L)$ satisfies certain monotonicity properties. In particular, if we interpret $v_{i, t}\left(r_{i}\right)$ as the value of having $r_{i}$ units of resource $i$ at time period $t$, then $v_{i, t}\left(r_{i}\right)-v_{i, t}\left(r_{i}-1\right)$ is the marginal value of capacity on resource $i$ at time period $t$. Part (i) of Lemma 1 shows that the marginal value of capacity is decreasing in $t$ keeping $r_{i}$ constant, while part (ii) shows that the marginal value of capacity is decreasing in $r_{i}$ for a given $t$.

These properties are quite natural and turn out to be useful for a couple of reasons. First, Lemma 1 implies that the optimal objective function value of $(P L)$ is not affected by adding constraints of the form $v_{i, t}\left(r_{i}\right)-v_{i, t}\left(r_{i}-1\right) \geq v_{i, t}\left(r_{i}+1\right)-v_{i, t}\left(r_{i}\right)$ to the linear program. This decreasing marginal value property turns out to be crucial in showing the polynomial-time solvability of the separation problem for the piecewise-linear approximation.

LEMma 1. There exists an optimal solution $\left\{\hat{v}_{i, t}\left(r_{i}\right) \mid \forall t, i, r_{i} \in \mathcal{R}_{i}\right\}$ to $(P L)$ such that

(i) $\hat{v}_{i, t}\left(r_{i}\right)-\hat{v}_{i, t}\left(r_{i}-1\right) \geq \hat{v}_{i, t+1}\left(r_{i}\right)-\hat{v}_{i, t+1}\left(r_{i}-1\right)$ for all $t, i$ and $r_{i} \in \mathcal{R}_{i}$,

(ii) $\hat{v}_{i, t}\left(r_{i}\right)-\hat{v}_{i, t}\left(r_{i}-1\right) \geq \hat{v}_{i, t}\left(r_{i}+1\right)-\hat{v}_{i, t}\left(r_{i}\right)$ for all $t, i$, and $r_{i} \in \mathcal{R}_{i}$, where we define $\hat{v}_{i, t}\left(r_{i}^{1}+1\right)=\hat{v}_{i, t}\left(r_{i}^{1}\right)$ for all $t$ and $i$.

$A$ ppendix. Q.E.D.

2.4. Lagrangian Relaxation Topaloglu [20] proposes a Lagrangian relaxation approach that decomposes the network RM problem into a number of single-resource problems by decoupling the acceptance decisions for a product over the resources that it uses via product and time-specific Lagrange multipliers. The idea is to write the network RM problem in terms of resource-level controls and then relax the constraints that link the resource-level decisions together. Letting $\boldsymbol{u}^{i t}=\left[u_{j}^{i t}\right]$ represent a control vector associated with resource $i$ at time $t$, with $u_{j}^{i t} \in\{0,1\}$ representing the acceptance decision on resource $i-1$ if we accept product $j$ on resource $i$ at time $t$ and 0 otherwise, the network RM problem requires that the resource-level decisions $u_{j}^{i t}$ be coordinated across all resources $i \in \mathcal{I}_{j}$. That is, either $u_{j}^{i t}=0$ for all $i \in \mathcal{I}_{j}$ or $u_{j}^{i t}=1$ for all $i \in \mathcal{I}_{j}$. Topaloglu [20] relaxes the constraints that link the resourcelevel decisions by associating Lagrange multipliers with them.

Let $\left\{\lambda_{i, j, t} \mid \forall t, j, i \in \mathcal{I}_{j}\right\}$ denote a set of Lagrange multipliers and

$$
\mathcal{U}_{i}\left(r_{i}\right)=\left\{\boldsymbol{u}^{i} \in\{0,1\}^{n} \mid u_{j}^{i} \leq r_{i}, \forall j \in \mathcal{J}_{i}\right\}
$$

denote the set of feasible controls on resource $i$ when its capacity is $r_{i}$. We solve the optimality equation

$$
\vartheta_{i, t}^{\lambda}\left(r_{i}\right)=\max _{\boldsymbol{u}^{i} \in \mathcal{U}_{i}\left(r_{i}\right)} \sum_{j \in \mathcal{J}_{i}} p_{j, t} u_{j}^{i}\left[\lambda_{i, j, t}+\vartheta_{i, t+1}^{\lambda}\left(r_{i}-1\right)-\vartheta_{i, t+1}^{\lambda}\left(r_{i}\right)\right]+\vartheta_{i, t+1}^{\lambda}\left(r_{i}\right)
$$

for resource $i$, with the boundary condition $\vartheta_{i, \tau+1}^{\lambda}(\cdot)=0$. We note that $\vartheta_{i, t}^{\lambda}(\cdot)$ can be interpreted as the value function associated with a single resource RM problem where $\left\{\lambda_{i, j, t} \mid \forall t, j \in \mathcal{J}_{i}\right\}$ are the revenues of the products.

Collecting the single resource value functions together, we let

$$
V_{t}^{\lambda}(\boldsymbol{r})=\sum_{t^{\prime}=t}^{\tau} \sum_{j} p_{j, t^{\prime}}\left[f_{j}-\sum_{i \in \mathcal{I}_{j}} \lambda_{i, j, t^{\prime}}\right]^{+}+\sum_{i} \vartheta_{i, t}^{\lambda}\left(r_{i}\right) .
$$

We can interpret $V_{t}^{\lambda}(\boldsymbol{r})$ as an approximation to $V_{t}(\boldsymbol{r})$ and indeed it is possible to show that $V_{t}^{\lambda}(\boldsymbol{r})$ is an upper bound on $V_{t}(\boldsymbol{r})$, where we use $[x]^{+}=\max \{0, x\}$. The Lagrangian relaxation approach finds the tightest upper bound on the optimal expected revenue by solving

$$
V^{L R}=\min _{\lambda} V_{1}^{\lambda}\left(\boldsymbol{r}^{1}\right)
$$

Talluri [16] shows that the optimal Lagrange multipliers satisfy $\sum_{i \in \mathcal{I}_{j}} \lambda_{i, j, t}=f_{j}$ for all $j$ and $t$. 
Proposition 1. There exists $\left\{\hat{\lambda}_{i, j, t} \mid \forall t, j, i \in \mathcal{I}_{j}\right\} \in \arg \min _{\lambda} V_{1}^{\lambda}\left(\boldsymbol{r}^{1}\right)$ that satisfy $\hat{\lambda}_{i, j, t} \geq 0$ and $\sum_{i \in \mathcal{I}_{j}} \hat{\lambda}_{i, j, t}=f_{j}$ for all $j$ and $t$.

$A$ ppendix. Q.E.D.

Proposition 1 implies that we can find the optimal Lagrange multipliers by solving

$$
V^{L R}=\min _{\left\{\lambda \mid \sum_{i \in \mathcal{I}_{j}} \lambda_{i, j, t}=f_{j}, \lambda_{i, j, t} \geq 0, \forall t, j, i \in \mathcal{I}_{j}\right\}} \sum_{i} \vartheta_{i, 1}^{\lambda}\left(r_{i}^{1}\right) .
$$

Using Proposition 1, we can interpret the Lagrange multiplier $\lambda_{i, j, t}$ as the portion of the revenue associated with product $j$ that we allocate to resource $i$ at time period $t$. With this understanding $\vartheta_{i, 1}^{\lambda}\left(r_{i}^{1}\right)$ is the value function of a single-resource $\mathrm{RM}$ problem with revenues $\left\{\lambda_{i, j, t} \mid \forall j \in \mathcal{J}_{i}, t\right\}$ on resource $i$. Therefore, we can also obtain the optimal Lagrange multipliers through the linear programming formulation of the single-resource RM dynamic program, with a set of linking constraints (5) as below:

$$
\begin{aligned}
V^{L R}=\min _{\lambda, \nu} & \sum_{i} \nu_{i, 1}\left(r_{i}^{1}\right) \\
\text { s.t. } & \begin{array}{c}
\nu_{i, t}\left(r_{i}\right) \geq \sum_{j \in \mathcal{J}_{i}} p_{j, t} u_{j}^{i}\left[\lambda_{i, j, t}+\nu_{i, t+1}\left(r_{i}-1\right)-\nu_{i, t+1}\left(r_{i}\right)\right] \\
\quad \nu_{i, t+1}\left(r_{i}\right) \quad \forall t, i, r_{i} \in \mathcal{R}_{i}, \boldsymbol{u}^{i} \in \mathcal{U}_{i}\left(r_{i}\right)
\end{array} \\
& \sum_{i \in \mathcal{I}_{j}} \lambda_{i, j, t}=f_{j} \quad \forall t, j \\
& \lambda_{i, j, t} \geq 0 \forall t, j, i \in \mathcal{I}_{j} ; \nu_{i, \tau+1}(\cdot)=0 \forall i .
\end{aligned}
$$

The linear programming formulation $(L R)$ turns out to be useful when comparing the Lagrangian relaxation approach with the piecewise-linear approximation.

3. Polynomial-time solvability of the piecewise-linear approximation In this section we show that the linear programming formulation of the piecewise-linear approximation is separable in polynomial time. A byproduct of our result is that the Lagrangian relaxation is equivalent to the piecewise-linear approximation, in that they yield the same upper bound on the value function.

Proposition 2. $V^{P L}=V^{L R}$.

It is easy to see that $V^{P L} \leq V^{L R}$ since $(L R)$ gives a separable approximation that is an upper bound, while $(P L)$ gives the tightest separable approximation that is an upper bound; we give a formal proof in §3.3. So the difficult part is the other direction: In the Lagrangian problem, we solve each of the resources independently and a product might be accepted on one resource and rejected on another, and there is no reason to believe that the Lagrange multipliers co-ordinate perfectly-indeed there are few known dynamic programs where they do. For the network RM problem, Proposition 4 below shows that there exists a set of Lagrange multipliers that perfectly coordinate the acceptance decisions across the resources. As a result, we can use these Lagrange multipliers to disaggregate constraint (2) of $(P L)$ into $|\mathcal{I}|$ constraints, one for each resource. The disaggregated resource level constraints essentially have the same form as constraints (4) of $(L R)$, which in turn can be used to show that $V^{L R} \leq V^{P L}$.

In $\S 3.1$ we first set up the separation problem for $(P L)$, a simpler alternative to solving $(P L)$ directly. We then show that constraints $(2)$ in $(P L)$ can be separated by solving a Lagrangian relaxation (with a linear programming representation). We describe a polynomial-time separation algorithm for $(P L)$ in $\S 3.2$. We build on these results and give a formal proof of Proposition 2 in $\S 3.3$ by lifting this separation linear program.

3.1. Separation for $(P L)$ Since $(P L)$ has an exponential number of constraints of type $(2)$, we use a separation algorithm to solve $(P L)$. Equivalence of efficient separation and solvability of a linear program is due to the well-known work of Grötschel et al. [7]. 
The idea is to start with a linear program that has a small subset of constraints (2) and solve it to obtain $\overline{\mathcal{V}}=\left\{\bar{v}_{i, t}\left(r_{i}\right) \mid \forall t, i, r_{i} \in \mathcal{R}_{i}\right\}$. We then check for violated constraints by solving the following separation problem: Prove that $\overline{\mathcal{V}}$ satisfies all the constraints (2), and if not, find a violated constraint and add it to the linear program. Throughout we assume that $\overline{\mathcal{V}}$ satisfies $\bar{v}_{i, t}\left(r_{i}\right)-\bar{v}_{i, t}\left(r_{i}-1\right) \geq \bar{v}_{i, t}\left(r_{i}+\right.$ 1) $-\bar{v}_{i, t}\left(r_{i}\right)$ for all $t, i$ and $r_{i} \in \mathcal{R}_{i}$. This is without loss of generality, since by Lemma 1 , we can add these constraints to $(P L)$ without affecting its optimal objective function value.

Let $\Delta_{i, t}\left(r_{i}\right)=\bar{v}_{i, t+1}\left(r_{i}\right)-\bar{v}_{i, t}\left(r_{i}\right)$ and $\psi_{i, t}\left(r_{i}\right)=\bar{v}_{i, t}\left(r_{i}\right)-\bar{v}_{i, t}\left(r_{i}-1\right)$ for $r_{i} \in \mathcal{R}_{i}$, so that the separation problem for $(P L)$ for period $t$ can be written as

$$
\Phi_{t}(\overline{\mathcal{V}})=\max _{\boldsymbol{r} \in \mathcal{R}, \boldsymbol{u} \in \mathcal{U}(\boldsymbol{r})} \sum_{j} p_{j, t} u_{j}\left[f_{j}-\sum_{i \in \mathcal{I}_{j}} \psi_{i, t+1}\left(r_{i}\right)\right]+\sum_{i} \Delta_{i, t}\left(r_{i}\right) .
$$

Note that $\psi_{i, t}(\cdot)$ is just the marginal value of capacity on resource $i$ at time period $t$. The separation problem for a set of values $\overline{\mathcal{V}}$ is resolved by obtaining the value of $\Phi_{t}(\overline{\mathcal{V}})$ and checking if for any $t$, $\Phi_{t}(\overline{\mathcal{V}})>0$.

By Lemma 1, $\psi_{i, t}\left(r_{i}\right)$ is nonincreasing in $r_{i}$. By definition, $\bar{v}_{i, t}\left(r_{i}^{1}+1\right)=\bar{v}_{i, t}\left(r_{i}^{1}\right)$. Since $\psi_{i, t}\left(r_{i}^{1}\right)=$ $\bar{v}_{i, t}\left(r_{i}^{1}\right)-\bar{v}_{i, t}\left(r_{i}^{1}-1\right) \geq \bar{v}_{i, t}\left(r_{i}^{1}+1\right)-\bar{v}_{i, t}\left(r_{i}^{1}\right)=0$, we also have $\psi_{i, t}\left(r_{i}\right) \geq 0$ for all $r_{i} \in \mathcal{R}_{i}$. We show that problem (6) can be solved efficiently as a linear program. This result is useful for two reasons. First, it helps us in establishing the equivalence between the piecewise linear approximation and the Lagrangian relaxation approaches. Second, it shows that separation can be efficiently carried out for $(P L)$.

We begin by describing a relaxation of problem (6) that decomposes it into a number of single resource problems. For time period $t$, we split the revenue of product $j, f_{j}$, among the resources that it consumes using variables $\lambda_{i, j, t}$, so that $\lambda_{i, j, t}$ represents the revenue allocated to resource $i \in \mathcal{I}_{j}$ at time $t$. Naturally, we have $\sum_{i \in \mathcal{I}_{j}} \lambda_{i, j, t}=f_{j}$ and $\lambda_{i, j, t} \geq 0$ for all $i \in \mathcal{I}_{j}$ and for all $j$. Following Topaloglu [20], we can alternatively interpret $\left\{\lambda_{i, j, t} \mid \forall i \in \mathcal{I}_{j}\right\}$ as Lagrange multipliers associated with the constraints that link the acceptance decisions for product $j$ across the resources that it consumes. Given such a set of Lagrange multipliers, we solve the problem

$$
\Pi_{i, t}^{\lambda}(\overline{\mathcal{V}})=\max _{r_{i} \in \mathcal{R}_{i}, \boldsymbol{u}^{i} \in \mathcal{U}_{i}\left(r_{i}\right)} \sum_{j \in \mathcal{J}_{i}} p_{j, t} u_{j}^{i}\left[\lambda_{i, j, t}-\psi_{i, t+1}\left(r_{i}\right)\right]+\Delta_{i, t}\left(r_{i}\right)
$$

for each resource $i$. The following lemma states that $\sum_{i} \Pi_{i, t}^{\lambda}(\overline{\mathcal{V}})$ is an upper bound on $\Phi_{t}(\overline{\mathcal{V}})$.

LEMma 2. If $\left\{\lambda_{i, j, t} \mid \forall t, j, i \in \mathcal{I}_{j}\right\}$ satisfy $\sum_{i \in \mathcal{I}_{j}} \lambda_{i, j, t}=f_{j}$ and $\lambda_{i, j, t} \geq 0$ for all $t, j$ and $i \in \mathcal{I}_{j}$, then $\Phi_{t}(\overline{\mathcal{V}}) \leq \sum_{i} \Pi_{i, t}^{\lambda}(\overline{\mathcal{V}})$.

If $\left(\boldsymbol{r}=\left[r_{i}\right], \boldsymbol{u} \in \mathcal{U}(\boldsymbol{r})\right)$ is optimal for problem (6), then $\boldsymbol{u} \in \mathcal{U}_{i}\left(r_{i}\right)$ and consequently $\left(r_{i}, \boldsymbol{u}\right)$ is feasible for problem (7). Q.E.D.

We next show that the upper bound is tight. That is, letting

$$
\Pi_{t}(\overline{\mathcal{V}})=\min _{\left\{\lambda \mid \sum_{i \in \mathcal{I}_{j}} \lambda_{i, j, t}=f_{j} \forall j ; \lambda_{i, j, t} \geq 0 \forall j, i \in \mathcal{I}_{j}\right\}} \sum_{i} \Pi_{i, t}^{\lambda}(\overline{\mathcal{V}})
$$

we have the following proposition.

Proposition 3. $\Phi_{t}(\overline{\mathcal{V}})=\Pi_{t}(\overline{\mathcal{V}})$.

Before we give a formal proof, we provide some intuition as to why the result holds. The equivalence of $\Phi_{t}(\overline{\mathcal{V}})$ and $\Pi_{t}(\overline{\mathcal{V}})$ turns out to be the key result in showing the equivalence between the piecewise-linear and the Lagrangian relaxation approaches.

3.1.1. Intuition behind Proposition 3 Lemma 2 shows that $\Phi_{t}(\overline{\mathcal{V}}) \leq \Pi_{t}(\overline{\mathcal{V}})$. So we only give a heuristic argument for why $\Phi_{t}(\overline{\mathcal{V}}) \geq \Pi_{t}(\overline{\mathcal{V}})$. Consider problem $(6)$. Noting that $\psi_{i, t+1}(0)=\infty$, an optimal solution will have $u_{j}=1$ only if the difference $f_{j}-\sum_{i \in \mathcal{I}_{j}} \psi_{i, t+1}\left(r_{i}\right)>0$. Therefore, we can write $u_{j}\left[f_{j}-\sum_{i \in \mathcal{I}_{j}} \psi_{i, t+1}\left(r_{i}\right)\right]$ in the objective function as $\left[f_{j}-\sum_{i \in \mathcal{I}_{j}} \psi_{i, t+1}\left(r_{i}\right)\right]^{+}$. Next, recall that $\psi_{i, t+1}(\cdot)$ is a decreasing function of $r_{i}$. Assuming it to be invertible (say it is strictly decreasing), we can write 
problem (6) with $\psi_{i, t+1}$ 's as the decision variables instead of the $r_{i}$ 's. Therefore, we can write a relaxed continuous version of problem (6) as

$$
\bar{\Phi}_{t}(\overline{\mathcal{V}})=\max _{\psi} \sum_{j} p_{j, t}\left[f_{j}-\sum_{i \in \mathcal{I}_{j}} \psi_{i, t+1}\right]^{+}+\sum_{i} \Delta_{i, t}\left(\psi_{i, t+1}\right) .
$$

The above problem is not differentiable. However, by smoothing the $[\cdot]^{+}$operator and assuming that $\Delta_{i, t}(\cdot)$ is differentiable, we can solve a differentiable problem which is arbitrarily close to problem (9). So we can assume that an optimizer of the above maximization problem $\left\{\hat{\psi}_{i, t+1} \mid \forall i\right\}$ satisfies the first order condition

$$
-\sum_{j \in \mathcal{J}_{i}} p_{j, t} \mathbb{1}_{\left[f_{j}-\sum_{k \in \mathcal{I}_{j}} \hat{\psi}_{k, t+1}>0\right]}+\Delta_{i, t}^{\prime}\left(\hat{\psi}_{i, t+1}\right)=0
$$

for all $i$, where $\Delta_{i, t}^{\prime}(\cdot)$ denotes the derivative of $\Delta_{i, t}\left(\psi_{i, t+1}\right)$ with respect to $\psi_{i, t+1}$. We emphasize that the above arguments are heuristic; our goal here is to only give intuition.

We use the optimal solution $\left\{\hat{\psi}_{i, t+1} \mid \forall i\right\}$ described above to construct a set of Lagrange multipliers in the following manner. Let

$$
\hat{\lambda}_{i, j, t}=f_{j} \frac{\hat{\psi}_{i, t+1}}{\sum_{k \in \mathcal{I}_{j}} \hat{\psi}_{k, t+1}} \forall j, i \in \mathcal{I}_{j},
$$

and note that they are feasible to problem (8). We have

$$
\hat{\lambda}_{i, j, t}-\hat{\psi}_{i, t+1}=\left[f_{j}-\sum_{k \in \mathcal{I}_{j}} \hat{\psi}_{k, t+1}\right] \frac{\hat{\psi}_{i, t+1}}{\sum_{k \in \mathcal{I}_{j}} \hat{\psi}_{k, t+1}} .
$$

Since the ratio on the right hand side is positive, this implies $\mathbb{1}_{\left[\hat{\lambda}_{i, j, t}-\hat{\psi}_{i, t+1}>0\right]}=\mathbb{1}_{\left[f_{j}-\sum_{k \in \mathcal{I}_{j}} \hat{\psi}_{k, t+1}>0\right]}$. Since, $\left\{\hat{\psi}_{i, t+1} \mid \forall i\right\}$ satisfies (10), it also satisfies

$$
-\sum_{j \in \mathcal{J}_{i}} p_{j, t} \mathbb{1}_{\left[\hat{\lambda}_{i, j, t}-\hat{\psi}_{i, t+1}>0\right]}+\Delta_{i, t}^{\prime}\left(\hat{\psi}_{i, t+1}\right)=0
$$

which is the first order condition associated with an optimizer of $\max _{\psi} \sum_{j \in \mathcal{J}_{i}} p_{j, t}\left[\hat{\lambda}_{i, j, t}-\psi_{i, t+1}\right]^{+}+$ $\Delta_{i, t}\left(\psi_{i, t+1}\right)$. Examining (12) we see that the first term is always negative and decreasing so the slope of $\Delta, \Delta^{\prime}$ has to be positive at this point. But the term $\sum_{j \in \mathcal{J}_{i}} p_{j, t}\left[\hat{\lambda}_{i, j, t}-\psi_{i, t+1}\right]^{+}$is decreasing in $\psi$, so the $\Delta$ can cross it with positive slope only once. That is, $\left\{\hat{\psi}_{i, t+1} \mid \forall i\right\}$ is an optimizer of $\max _{\psi} \sum_{j \in \mathcal{J}_{i}} p_{j, t}\left[\hat{\lambda}_{i, j, t}-\right.$ $\left.\psi_{i, t+1}\right]^{+}+\Delta_{i, t}\left(\psi_{i, t+1}\right)$.

So a relaxed continuous version of problem (7) can be written as

$$
\bar{\Pi}_{i, t}^{\hat{\lambda}}(\overline{\mathcal{V}})=\max _{\psi} \sum_{j \in \mathcal{J}_{i}} p_{j, t}\left[\hat{\lambda}_{i, j, t}-\psi_{i, t+1}\right]^{+}+\Delta_{i, t}\left(\psi_{i, t+1}\right)=\sum_{j \in \mathcal{J}_{i}} p_{j, t}\left[\hat{\lambda}_{i, j, t}-\hat{\psi}_{i, t+1}\right]^{+}+\Delta_{i, t}\left(\hat{\psi}_{i, t+1}\right),
$$

where the last equality follows from above observations.

Putting everything together, we have

$$
\begin{array}{r}
\Pi_{t}(\overline{\mathcal{V}}) \leq \sum_{i} \bar{\Pi}_{i, t}^{\hat{\lambda}}(\overline{\mathcal{V}})=\sum_{i}\left\{\sum_{j \in \mathcal{J}_{i}} p_{j, t}\left[\hat{\lambda}_{i, j, t}-\hat{\psi}_{i, t+1}\right]^{+}+\Delta_{i, t}\left(\hat{\psi}_{i, t+1}\right)\right\} \\
=\sum_{j} p_{j, t} \sum_{i \in \mathcal{I}_{j}}\left[\hat{\lambda}_{i, j, t}-\hat{\psi}_{i, t+1}\right]^{+}+\sum_{i} \Delta_{i, t}\left(\hat{\psi}_{i, t+1}\right)=\bar{\Phi}_{t}(\overline{\mathcal{V}})
\end{array}
$$

where the first inequality holds since $\left\{\hat{\lambda}_{i, j, t} \mid \forall j, i \in \mathcal{I}_{j}\right\}$ is feasible for problem (8) and the last equality uses (11) and the fact that $\left\{\hat{\psi}_{i, t+1} \mid \forall i\right\}$ is optimal for (9). Note also that the Lagrange multipliers 
$\left\{\hat{\lambda}_{i, j, t} \mid \forall j, i \in, j\right\}$ coordinate the decisions for each product across the different resources: product $j$ is accepted on resource $i \in \mathcal{I}_{j}$ only if $\hat{\lambda}_{i, j, t}-\hat{\psi}_{i, t+1}>0$. By (11), either $\hat{\lambda}_{i, j, t}-\hat{\psi}_{i, t+1}>0$ for all $i \in \mathcal{I}_{j}$ or $\hat{\lambda}_{i, j, t}-\hat{\psi}_{i, t+1} \leq 0$ for all $i \in \mathcal{I}_{j}$. That is, we either accept the product on all the resources it consumes or reject the product on all the resources it consumes.

Fixing the Lagrange multipliers as $\hat{\lambda}$, we claim this is an optimal solution to (7) that happens at an integer value of $r_{i}$. Note that (7) is composed of two piecewise-linear functions, the $\Delta$ function (that in the continuous relaxation is interpolated linearly) that has breakpoints only at integer $r_{i}$ and the first part which as $r_{i}$ increases has $\psi_{i, t+1}\left(r_{i}\right)$ decreasing, and hence $\left[\hat{\lambda}_{i, j, t}-\psi_{i, t+1}\left(r_{i}\right)\right]^{+}$increasing with increasing $r_{i}$. If we decrease $\psi$ continuously in $\sum_{j \in \mathcal{J}_{i}} p_{j, t}\left[\hat{\lambda}_{i, j, t}-\psi\right]^{+}$, we again obtain a continuous piecewise-linear function with possible non-integer breakpoints that moreover has increasing slope after each new breakpoint (i.e. is convex between the integer breakpoints). So between two integers $r_{i}$, as it is a sum of a linear function and a piece-wise linear convex function, the maximizer to the one-dimensional relaxed problem $\max _{\psi_{i, t+1}} \sum_{j \in \mathcal{J}_{i}} p_{j, t}\left[\hat{\lambda}_{i, j, t}-\psi_{i, t+1}\right]^{+}+\Delta_{i, t}\left(\psi_{i, t+1}\right)$ should happen at an integer $r_{i}$. We therefore have $\bar{\Phi}_{t}(\overline{\mathcal{V}})=\Phi_{t}(\overline{\mathcal{V}})=\Pi_{t}(\overline{\mathcal{V}})$ if the optimal $\psi_{i, t+1}^{-1}(\hat{\psi})$ is an integer.

We once again emphasize that the above arguments are heuristic; we give a formal proof in the following section that is quite distinct from the above reasoning.

3.1.2. Proof of Proposition 3 We begin with some preliminary results. First, we show that problem (7) can be written as the following linear program

$$
\begin{aligned}
\Pi_{i, t}^{\lambda}(\overline{\mathcal{V}})=\min _{w, z} & w_{i, t} \\
\text { s.t. } & \\
& w_{i, t} \geq \sum_{j \in \mathcal{J}_{i}} z_{i, j, t, r}+\Delta_{i, t}(r) \quad \forall r \in \mathcal{R}_{i} \\
& z_{i, j, t, r} \geq p_{j, t}\left[\lambda_{i, j, t}-\psi_{i, t+1}(r)\right] \quad \forall j \in \mathcal{J}_{i}, r \in \mathcal{R}_{i} \\
& z_{i, j, t, r} \geq 0 \forall j \in \mathcal{J}_{i}, r \in \mathcal{R}_{i} .
\end{aligned}
$$

Lemma 3. The linear program $\left(S e p L R_{i}\right)$ is equivalent to $(7)$.

$A$ ppendix. Q.E.D.

We can, therefore, formulate problem (8) as the linear program

$$
\begin{aligned}
\Pi_{t}(\overline{\mathcal{V}})=\min _{\lambda, w, z} & \sum_{i} w_{i, t} \\
\text { s.t. } \quad & \\
& w_{i, t} \geq \sum_{j \in \mathcal{J}_{i}} z_{i, j, t, r}+\Delta_{i, t}(r) \quad \forall i, r \in \mathcal{R}_{i} \\
& z_{i, j, t, r} \geq p_{j, t}\left[\lambda_{i, j, t}-\psi_{i, t+1}(r)\right] \quad \forall i, j \in \mathcal{J}_{i}, r \in \mathcal{R}_{i} \\
& \sum_{i \in \mathcal{I}_{j}} \lambda_{i, j, t}=f_{j} \quad \forall j \\
& \lambda_{i, j, t} \geq 0 \quad \forall i, j \in \mathcal{J}_{i} \\
& z_{i, j, t, r} \geq 0 \quad \forall i, j \in \mathcal{J}_{i}, r \in \mathcal{R}_{i} .
\end{aligned}
$$

With a slight abuse of notation, we let $(\lambda, w, z)=\left(\left\{\lambda_{i, j, t} \mid \forall j, i \in \mathcal{I}_{j}\right\},\left\{w_{i, t} \mid \forall i\right\},\left\{z_{i, j, t, r} \mid \forall i, j \in \mathcal{J}_{i}, r \in \mathcal{R}_{i}\right\}\right)$ denote a feasible solution to $(\operatorname{Sep} L R)$. Let $\xi_{i, t}(r)=w_{i, t}-\left[\sum_{j \in \mathcal{J}_{i}} z_{i, j, t, r}+\Delta_{i, t}(r)\right]$ denote the slack in constraint (14), and $B_{i, t}(\lambda, w, z)=\left\{r \in \mathcal{R}_{i} \mid \xi_{i, t}(r)=0\right\}$ denote the set of binding constraints of type (14) and $B_{i, t}^{c}(\lambda, w, z)$ denote its complement.

Note that if $(\hat{\lambda}, \hat{w}, \hat{z})$ is an optimal solution, then $B_{i, t}(\hat{\lambda}, \hat{w}, \hat{z})$ is nonempty, since for each resource $i$, there exists some $r_{i} \in \mathcal{R}_{i}$ such that constraint (14) is satisfied as an equality.

The following proposition is a key result. It says that there exists a set of optimal Lagrange multipliers that perfectly coordinate the acceptance decisions for each product across all the resources. That is, even though we solve the single resource problems in a decentralized fashion, the Lagrange multipliers are such that we either accept the product on all the resources or reject it on all the resources. 
We say that $(\hat{\lambda}, \hat{w}, \hat{z})$ is an optimal solution to $($ SepLR) with a minimal set of binding constraints $\bigcup_{i \in \mathcal{I}} B_{i, t}(\hat{\lambda}, \hat{w}, \hat{z})$, if there is no other optimal solution $\left(\hat{\lambda}^{\prime}, \hat{w}^{\prime}, \hat{z}^{\prime}\right)$ which has a set of binding constraints that is a strict subset of the binding constraints of $(\hat{\lambda}, \hat{w}, \hat{z})$; that is,

$$
\bigcup_{i \in \mathcal{I}} B_{i, t}\left(\hat{\lambda}^{\prime}, \hat{w}^{\prime}, \hat{z}^{\prime}\right) \subsetneq \bigcup_{i \in \mathcal{I}} B_{i, t}(\hat{\lambda}, \hat{w}, \hat{z})
$$

Proposition 4. There exists an optimal solution $(\hat{\lambda}, \hat{w}, \hat{z})$ to $(\operatorname{Sep} L R)$ with a minimal set of binding constraints and a vector $\hat{\boldsymbol{r}}$ with $\hat{r}_{i} \in B_{i, t}(\hat{\lambda}, \hat{w}, \hat{z})$ for all $i$, such that for each $j$, we either have $\hat{\lambda}_{i, j, t} \leq$ $\psi_{i, t+1}\left(\hat{r}_{i}\right)$ for all $i \in \mathcal{I}_{j}$ or $\hat{\lambda}_{i, j, t} \geq \psi_{i, t+1}\left(\hat{r}_{i}\right)$ for all $i \in \mathcal{I}_{j}$.

$A$ ppendix. Q.E.D.

We remark that Proposition 4 formalizes the intuition that there exists a set of optimal Lagrange multipliers that perfectly coordinate the acceptance decisions for each product across the resources it consumes. Indeed, if for product $j$, we have $\hat{\lambda}_{i, j, t} \leq \psi_{i, t+1}\left(\hat{r}_{i}\right)$ for all $i \in \mathcal{I}_{j}$, then $f_{j}=\sum_{i \in \mathcal{I}_{j}} \hat{\lambda}_{i, j, t} \leq$ $\sum_{i \in \mathcal{I}_{j}} \psi_{i, t+1}\left(\hat{r}_{i}\right)$. Therefore, $\left.\mathbb{1}_{\left[\hat{\lambda}_{i, j, t}-\psi_{i, t+1}\left(\hat{r}_{i}\right)>0\right]}=\mathbb{1}_{\left[f_{j}-\sum_{k \in \mathcal{I}_{j}} \psi_{k, t+1}\left(\hat{r}_{k}\right)>0\right.}\right]$. An implication of this, as the following arguments show, is that the acceptance decisions for product $j$ are exactly the same for all $i \in \mathcal{I}_{j}$.

By Lemma $2, \Phi_{t}(\overline{\mathcal{V}}) \leq \Pi_{t}(\overline{\mathcal{V}})$. We show below that $\Phi_{t}(\overline{\mathcal{V}}) \geq \Pi_{t}(\overline{\mathcal{V}})$, which completes the proof. Let $(\hat{\lambda}, \hat{w}, \hat{z})$ and $\left\{\hat{r}_{i} \mid \forall i\right\}$ be as in Proposition 4 ; so we have

$$
\Pi_{t}(\overline{\mathcal{V}})=\sum_{i} \hat{w}_{i, t}=\sum_{i} \sum_{j \in \mathcal{J}_{i}} \hat{z}_{i, j, t, \hat{r}_{i}}+\Delta_{i, t}\left(\hat{r}_{i}\right)=\sum_{j} \sum_{i \in \mathcal{I}_{j}} p_{j, t}\left[\hat{\lambda}_{i, j, t}-\psi_{i, t+1}\left(\hat{r}_{i}\right)\right]^{+}+\sum_{i} \Delta_{i, t}\left(\hat{r}_{i}\right)
$$

where the second equality holds since $\hat{r}_{i} \in B_{i, t}(\hat{\lambda}, \hat{w}, \hat{z})$ for all $i$. The last equality holds since if $\hat{z}_{i, j, t, \hat{r}_{i}}>$ $p_{j, t}\left[\hat{\lambda}_{i, j, t}-\psi_{i, t+1}\left(\hat{r}_{i}\right)\right]^{+}$, then we can decrease $\hat{z}_{i, j, t, \hat{r}_{i}}$ by a small positive number contradicting either the optimality of $(\hat{\lambda}, \hat{w}, \hat{z})$ or the fact that $(\hat{\lambda}, \hat{w}, \hat{z})$ is an optimal solution with a minimal set of binding constraints amongst all optimal solutions. Let $\mathcal{J}_{1}=\left\{j \mid \hat{\lambda}_{i, j, t} \geq \psi_{i, t+1}\left(\hat{r}_{i}\right) \forall i \in \mathcal{I}_{j}\right\}$ and $\mathcal{J}_{2}=\mathcal{J} \backslash \mathcal{J}_{1}$ where $\mathcal{J}=\{1, \ldots, n\}$. By Proposition 4, every product $j \in \mathcal{J}_{2}$ satisfies $\hat{\lambda}_{i, j, t} \leq \psi_{i, t+1}\left(\hat{r}_{i}\right)$ for all $i \in \mathcal{I}_{j}$. Therefore,

$$
\begin{aligned}
\Pi_{t}(\overline{\mathcal{V}}) & =\sum_{j \in \mathcal{J}_{1}} \sum_{i \in \mathcal{I}_{j}} p_{j, t}\left[\hat{\lambda}_{i, j, t}-\psi_{i, t+1}\left(\hat{r}_{i}\right)\right]+\sum_{i} \Delta_{i, t}\left(\hat{r}_{i}\right) \\
& =\sum_{j} \sum_{i \in \mathcal{I}_{j}} p_{j, t} \hat{u}_{j}\left[\hat{\lambda}_{i, j, t}-\psi_{i, t+1}\left(\hat{r}_{i}\right)\right]+\sum_{i} \Delta_{i, t}\left(\hat{r}_{i}\right) \\
& =\sum_{j} p_{j, t} \hat{u}_{j}\left[f_{j}-\sum_{i \in \mathcal{I}_{j}} \psi_{i, t+1}\left(\hat{r}_{i}\right)\right]+\sum_{i} \Delta_{i, t}\left(\hat{r}_{i}\right) \\
& \leq \Phi_{t}(\overline{\mathcal{V}})
\end{aligned}
$$

where we define $\hat{u}_{j}=1$ for $j \in \mathcal{J}_{1}$ and $\hat{u}_{j}=0$ for $j \in \mathcal{J}_{2}$. Note that the last equality follows from constraint (16). The last inequality holds since $\boldsymbol{r}=\left[\hat{r}_{i}\right], \boldsymbol{u}=\left[\hat{u}_{j}\right]$ is feasible to problem (6) by the following argument: we trivially have $\hat{u}_{j} \leq \hat{r}_{i}$ for all $j \in \mathcal{J}_{2}$ and $i \in \mathcal{I}_{j}$. On the other hand, since $\hat{\lambda}_{i, j, t}$ is finite and $\psi_{i, t+1}(0)=\infty$, we have $\hat{r}_{i} \geq 1$ for all $j \in \mathcal{J}_{1}$ and $i \in \mathcal{I}_{j}$. It follows that $\hat{u}_{j} \leq \hat{r}_{i}$ for all $j \in \mathcal{J}_{1}$ and $i \in \mathcal{I}_{j}$; so the 0-1 controls $\hat{\boldsymbol{u}}=\left\{\hat{u}_{j}\right\}$ satisfy the constraint that $\hat{u}_{j}=0$ if $\hat{r}_{i}=0$ for any $i \in \mathcal{I}_{j}$. Q.E.D.

3.2. Polynomial-time separation for $(P L)$ Proposition 3 implies that the separation for $(P L)$ can be done by solving the compact linear program $(S e p L R)$ for a given set of $\overline{\mathcal{V}}$ variables. If its optimal objective function value $\Pi_{t}(\overline{\mathcal{V}}) \leq 0$ for all $t$ then $\overline{\mathcal{V}}$ is feasible in $(P L)$. If $\Pi_{t}(\overline{\mathcal{V}})>0$ for some $t$, then we find a state-action pair $(\boldsymbol{r} \in \mathcal{R}, \boldsymbol{u} \in \mathcal{U}(\boldsymbol{r}))$ that violates constraint (2) in the following manner. 
Separation Algorithm: If $\Pi_{t}(\overline{\mathcal{V}}) \leq 0$ Then stop, Else:

Step 1: Let $\left(\hat{\lambda}^{(0)}, \hat{w}^{(0)}, \hat{z}^{(0)}\right)$ be an optimal solution to $(\operatorname{SepLR})$. Set $k=0$.

Step 2: Let $\left\{\hat{r}_{i}^{(k)} \mid \forall i\right\}$ be as defined in the proof of Proposition 4.

If, for all $j, \hat{\lambda}_{i, j, t}^{(k)} \leq \psi_{i, t+1}\left(\hat{r}_{i}^{(k)}\right)$ for all $i \in \mathcal{I}_{j}$ or $\hat{\lambda}_{i, j, t}^{(k)} \geq \psi_{i, t+1}\left(\hat{r}_{i}^{(k)}\right)$ for all $i \in \mathcal{I}_{j}$, set $u_{j}=1$ for all $j \in \mathcal{J}_{1}$ and $u_{j}=0$ for all $j \in \mathcal{J}_{2}$, where $\mathcal{J}_{1}$ and $\mathcal{J}_{2}$ are as defined in Proposition 3. Set $\boldsymbol{r}=\left\{\hat{r}_{i}^{(k)} \mid \forall i\right\}$ and $\boldsymbol{u}=\left\{u_{j} \mid \forall j\right\}$ and stop.

Else, pick a product $j$ such that for $i \in \mathcal{I}_{j}$, we have $\hat{\lambda}_{i, j, t}^{(k)}<\psi_{i, t+1}\left(\hat{r}_{i}^{(k)}\right)$, while for $l \in \mathcal{I}_{j}$, we have $\hat{\lambda}_{l, j, t}^{(k)}>\psi_{l, t+1}\left(\hat{r}_{l}^{(k)}\right)$. Let $\left(\bar{\lambda}^{(k)}, \bar{w}^{(k)}, \bar{z}^{(k)}\right)$ be as described in Proposition 4.

Step 3: Set $\hat{\lambda}^{(k+1)}=\bar{\lambda}^{(k)}, \hat{w}^{(k+1)}=\bar{w}^{(k)}$ and $\hat{z}^{(k+1)}=\bar{z}^{(k)}$. Set $k=k+1$ and go to Step 2.

PROPOSITION 5. The separation for $(P L)$ can be carried out in polynomial time.

$T$ he linear program $(S e p L R)$ can be solved in polynomial time. If $\Pi_{t}(\overline{\mathcal{V}}) \leq 0$, then Separation Algorithm terminates immediately and we are done. Otherwise, by Proposition $4,\left(\hat{\lambda}^{(k)}, \hat{w}^{(k)}, \hat{z}^{(k)}\right)$ is an optimal solution to $(S e p L R)$ for all $k$. Proposition 4 also implies that $\left(\hat{\lambda}^{(k+1)}, \hat{w}^{(k+1)}, \hat{z}^{(k+1)}\right)$ has strictly fewer number of binding constraints of type (14) than $\left(\hat{\lambda}^{(k)}, \hat{w}^{(k)}, \hat{z}^{(k)}\right)$. Since the number of binding constraints of type (14) in any optimal solution is at least $m$ and at most $\sum_{i} r_{i}^{1}$, Separation Algorithm terminates in polynomial time. Finally, by Proposition $3, \boldsymbol{u} \in \mathcal{U}(\boldsymbol{r})$. Therefore, we have obtained a state-action pair $(\boldsymbol{r}, \boldsymbol{u})$ that violates constraint $(2)$ in polynomial time. Q.E.D.

3.3. Proof of Proposition 2 We first show that $V^{P L} \leq V^{L R}$. Consider a feasible solution $\left(\left\{\hat{\lambda}_{i, j, t} \mid \forall t, j, i \in \mathcal{I}_{j}\right\},\left\{\hat{\nu}_{i, t}\left(r_{i}\right) \mid \forall t, i, r_{i} \in \mathcal{R}_{i}\right\}\right)$ to $(L R)$. For a given $t, \boldsymbol{r}=\left[r_{i}\right]$ and $\boldsymbol{u} \in \mathcal{U}(\boldsymbol{r})$, note that $\boldsymbol{u} \in \mathcal{U}_{i}\left(r_{i}\right)$ for all $i$. Summing up constraints (4) for $r_{i}$ and $\boldsymbol{u} \in \mathcal{U}_{i}\left(r_{i}\right)$ for all $i$,

$$
\begin{aligned}
\sum_{i} \hat{\nu}_{i, t}\left(r_{i}\right) & \geq \sum_{i} \sum_{j \in \mathcal{J}_{i}} p_{j, t} u_{j}\left[\hat{\lambda}_{i, j, t}+\hat{\nu}_{i, t+1}\left(r_{i}-1\right)-\hat{\nu}_{i, t+1}\left(r_{i}\right)\right]+\sum_{i} \hat{\nu}_{i, t+1}\left(r_{i}\right) \\
& =\sum_{j} p_{j, t} u_{j}\left[f_{j}+\sum_{i \in \mathcal{I}_{j}} \hat{\nu}_{i, t+1}\left(r_{i}-1\right)-\hat{\nu}_{i, t+1}\left(r_{i}\right)\right]+\sum_{i} \hat{\nu}_{i, t+1}\left(r_{i}\right)
\end{aligned}
$$

where the equality holds since $\sum_{i \in \mathcal{I}_{j}} \hat{\lambda}_{i, j, t}=f_{j}$. So $\left\{\hat{\nu}_{i, t}\left(r_{i}\right) \mid \forall t, i, r_{i} \in \mathcal{R}_{i}\right\}$ is a feasible solution to $(P L)$ with the same objective function value and we have $V^{P L} \leq V^{L R}$.

To show the reverse inequality, let $\underline{V}^{P L}=\min _{v} \sum_{i} v_{i, 1}\left(\overrightarrow{r_{i}^{1}}\right)+\sum_{t} \Pi_{t}(\mathcal{V})$, where $\mathcal{V}=\left\{v_{i, t}\left(r_{i}\right) \mid \forall t, i, r_{i} \in\right.$ $\left.\mathcal{R}_{i}\right\}$. We have

$$
V^{P L}=\min _{\left\{v \mid \Phi_{t}(\mathcal{V}) \leq 0\right\}} \sum_{i} v_{i, 1}\left(r_{i}^{1}\right)=\min _{\left\{v \mid \Pi_{t}(\mathcal{V}) \leq 0\right\}} \sum_{i} v_{i, 1}\left(r_{i}^{1}\right) \geq \min _{\left\{v \mid \Pi_{t}(\mathcal{V}) \leq 0\right\}}\left\{\sum_{i} v_{i, 1}\left(r_{i}^{1}\right)+\sum_{t} \Pi_{t}(\mathcal{V})\right\} \geq \underline{V}^{P L},
$$

where the first equality follows from (6) while the second one follows from Proposition 3. The first inequality follows since $\Pi_{t}(\mathcal{V})$ is constrained to be nonpositive, while the last equality uses the fact that $\underline{V}^{P L}$ does not have the constraints $\Pi_{t}(\mathcal{V}) \leq 0$.

Using (7) and the fact that $\Pi_{i, t}^{\lambda}(\mathcal{V})$ appears in the objective function of a minimization problem, we have

$$
\begin{array}{ll}
\underline{V}^{P L}= & \min _{\lambda, \pi, v} \sum_{t} \sum_{i} \pi_{i, t}+\sum_{i} v_{i, 1}\left(r_{i}^{1}\right) \\
\text { s.t. } & \pi_{i, t} \geq \sum_{j \in \mathcal{J}_{i}} p_{j, t} u_{j}^{i}\left[\lambda_{i, j, t}+v_{i, t+1}\left(r_{i}-1\right)-v_{i, t+1}\left(r_{i}\right)\right]+v_{i, t+1}\left(r_{i}\right)-v_{i, t}\left(r_{i}\right)
\end{array}
$$




$$
\begin{aligned}
& \sum_{i \in \mathcal{I}_{j}} \lambda_{i, j, t}=f_{j} \quad \forall t, j \quad \forall t, i, r_{i} \in \mathcal{R}_{i}, \boldsymbol{u}^{i} \in \mathcal{U}_{i}\left(r_{i}\right) \\
& \lambda_{i, j, t} \geq 0 \forall t, j, i \in \mathcal{I}_{j} ; v_{i, \tau+1}(\cdot)=0 \forall i .
\end{aligned}
$$

Letting $\pi_{i, t}=\theta_{i, t}-\theta_{i, t+1}$ with $\theta_{i, \tau+1}=0$, the above objective function becomes $\sum_{i} \theta_{i, 1}+v_{i, 1}\left(r_{i}^{1}\right)$ while the first set of constraints become $\theta_{i, t}+v_{i, t}\left(r_{i}\right) \geq \sum_{j \in \mathcal{J}_{i}} p_{j, t} u_{i, j}\left[\lambda_{i, j, t}+v_{i, t+1}\left(r_{i}-1\right)-v_{i, t+1}\left(r_{i}\right)\right]+\theta_{i, t+1}+$ $v_{i, t+1}\left(r_{i}\right)$. Finally, letting $\nu_{i, t}\left(r_{i}\right)=\theta_{i, t}+v_{i, t}\left(r_{i}\right)$, we have

$$
\begin{array}{ll}
\underline{V}^{P L} & =\min _{\lambda, \nu} \sum_{i} \nu_{i, 1}\left(r_{i}^{1}\right) \\
\text { s.t. } & \\
& \nu_{i, t}\left(r_{i}\right) \geq \sum_{j \in \mathcal{J}_{i}} p_{j, t} u_{j}^{i}\left[\lambda_{i, j, t}+\nu_{i, t+1}\left(r_{i}-1\right)-\nu_{i, t+1}\left(r_{i}\right)\right]+\nu_{i, t+1}\left(r_{i}\right) \\
& \sum_{i \in \mathcal{I}_{j}} \lambda_{i, j, t}=f_{j} \quad \forall t, j \quad \forall t, i, r_{i} \in \mathcal{R}_{i}, \boldsymbol{u}^{i} \in \mathcal{U}_{i}\left(r_{i}\right) \\
& \lambda_{i, j, t} \geq 0 \forall t, j, i \in \mathcal{I}_{j} ; \nu_{i, \tau+1}(\cdot)=0 \forall i .
\end{array}
$$

The above linear program is exactly $(L R)$, the linear programming formulation of the Lagrangian relaxation. So $V^{L R}=V^{P L} \leq V^{P L}$.

Therefore $V^{L R}=V^{\overline{P L}}$ as we argued the other, easier, direction earlier. Q.E.D.

4. Network RM with customer choice-importance of number of Lagrangian multipliers We consider the network RM problem with customer choice behavior. In choice-based RM, a customer chooses product $j$ with probability $p_{j}(S)$, when $S$ is the set of products offered. Note that $p_{j}(S)=0$ if $j \notin S$ and $1-\sum_{j} p_{j}(S)$ is the probability that the customer does not choose any of the offered products. Letting $\mathcal{Q}(\boldsymbol{r})=\left\{j \mid \mathbb{1}_{\left[j \in \mathcal{J}_{i}\right]} \leq r_{i} \forall i\right\}$ denote the set of products that can be offered given the resource capacities $\boldsymbol{r}$, the value functions $V_{t}(\cdot)$ can be obtained through the optimality equations

$$
V_{t}(\boldsymbol{r})=\max _{S \subset \mathcal{Q}(\boldsymbol{r})} \sum_{j} p_{j}(S)\left[f_{j}+V_{t+1}\left(\boldsymbol{r}-\sum_{i \in \mathcal{I}_{j}} \boldsymbol{e}^{i}\right)-V_{t+1}(\boldsymbol{r})\right]+V_{t+1}(\boldsymbol{r}),
$$

and the boundary condition is $V_{\tau+1}(\cdot)=0$.

The value functions can alternatively be obtained by solving the linear program

$$
\begin{aligned}
\min _{V_{t}(\cdot)} & V_{1}\left(\boldsymbol{r}^{1}\right) \\
\text { S.t } & \\
& V_{t}(\boldsymbol{r}) \geq \sum_{j} p_{j}(S)\left[f_{j}+V_{t+1}\left(\boldsymbol{r}-\sum_{i \in \mathcal{I}_{j}} \boldsymbol{e}^{i}\right)-V_{t+1}(\boldsymbol{r})\right]+V_{t+1}(\boldsymbol{r}) \\
& V_{\tau+1}(\cdot)=0 .
\end{aligned}
$$

Computing the value functions either through the optimality equations or the linear program is intractable. In the following sections, we describe extensions of the piecewise-linear and Lagrangian relaxation approaches to choice-based RM.

4.1. Piecewise-linear approximation The linear program from using a separable piecewiselinear approximation to the value function $V_{t}(\boldsymbol{r}) \approx \sum_{i} v_{i, t}\left(r_{i}\right)$ for all $\boldsymbol{r} \in \mathcal{R}$ is

$$
\begin{aligned}
& V^{C P L}=\min _{\substack{v \\
\text { s.t }}} \sum_{i} v_{i, 1}\left(r_{i}^{1}\right) \\
& \sum_{i} v_{i, t}\left(r_{i}\right) \geq \sum_{j} p_{j}(S)\left[f_{j}+\sum_{i \in \mathcal{I}_{j}}\left\{v_{i, t+1}\left(r_{i}-1\right)-v_{i, t+1}\left(r_{i}\right)\right\}\right] \\
& +\sum_{i} v_{i, t+1}\left(r_{i}\right) \quad \forall t, \boldsymbol{r} \in \mathcal{R}, S \subset \mathcal{Q}(\boldsymbol{r}) \\
& v_{i, \tau+1}(\cdot)=0 \quad \forall i .
\end{aligned}
$$


Meissner and Strauss [14] propose the piecewise-linear approximation for choice-based network RM. In order to make the formulation more tractable, they use an aggregation over the state variables to reduce to number of variables.

4.2. Lagrangian Relaxation A natural extension of the Lagrangian relaxation approach to the choice-based network RM problem is to use product and time-specific Lagrange multipliers $\left\{\lambda_{i, j, t} \mid \forall t, j, i \in \mathcal{I}_{j}\right\}$ to decompose the network problem into a number of single resource problems. Letting $\mathcal{Q}_{i}\left(r_{i}\right)=\left\{j \mid \mathbb{1}_{\left[j \in \mathcal{J}_{i}\right]} \leq r_{i}\right\}$, we solve the optimality equation

$$
\vartheta_{i, t}^{\lambda}\left(r_{i}\right)=\max _{S \subset \mathcal{Q}_{i}\left(r_{i}\right)} \sum_{j \in \mathcal{J}_{i}} p_{j}(S)\left[\lambda_{i, j, t}+\vartheta_{i, t+1}^{\lambda}\left(r_{i}-1\right)-\vartheta_{i, t+1}^{\lambda}\left(r_{i}\right)\right]+\vartheta_{i, t+1}^{\lambda}\left(r_{i}\right)
$$

for resource $i$. It is possible to show that $V_{t}^{\lambda}(\boldsymbol{r})=\sum_{i} \vartheta_{i, t}^{\lambda}\left(r_{i}\right)$ is an upper bound on $V_{t}(\boldsymbol{r})$. We find the tightest upper bound on the optimal expected revenue by solving

$$
V^{C L R}=\min _{\left\{\lambda \mid \lambda_{i, j, t} \geq 0, \sum_{i \in \mathcal{I}_{j}} \lambda_{i, j, t}=f_{j} \forall j, t\right\}} V_{1}^{\lambda}\left(\boldsymbol{r}^{1}\right) .
$$

In contrast to the independent demands setting, the Lagrangian relaxation turns out to be weaker than the piecewise-linear approximation; we give a simple example in the appendix which illustrates that we can have $V^{C P L}<V^{C L R}$. With customer choice, the decisions to the offer the different products get interlinked through the choice probabilities, and the product and time-specific Lagrange multipliers are not rich enough to ensure coordination of the offer sets across the resources. Kunnumkal and Talluri [10] propose a new Lagrangian relaxation approach using an expanded set of Lagrange multipliers that achieves equivalence to the piecewise-linear approximation. Therefore, the number and form of the Lagrange multipliers appear to be crucial to get the equivalence between the Lagrangian relaxation and the piecewise-linear approximation.

5. Numerical results As the separation problem for $(P L)$ is solvable in polynomial time, a plausible solution procedure is by linear programming, generating the constraints on the fly. In this section, we investigate how $(P L)$ compares $(L R)$. By our theoretical result both should give the same objective function value (as the numerical results indeed show), so our main interest is in comparing solution times.

We consider a hub and spoke network with a single hub that serves $N$ spokes. There is one flight from the hub to each spoke and one flight from each spoke to the hub. The total number of flights is $2 N$. Note that the flight legs correspond to the resources in our network RM formulation. We have a high fare-product and low fare-product connecting each origin-destination pair. Consequently, there are $2 N(N+1)$ fare-products in total. In all of our test problems, the high fare-product connecting an origin-destination pair is twice as expensive as the corresponding low fare-product. We measure the tightness of the flight leg capacities by

$$
\alpha=\frac{\sum_{i} \sum_{t} \sum_{j \in \mathcal{J}_{i}} p_{j, t}}{\sum_{i} r_{i}^{1}}
$$

where the numerator measures the total expected demand over the flight legs and the denominator gives the sum of the capacities of the flight legs. We index the test problems using $(\tau, N, \alpha)$, where $\tau$ is the number of periods in the booking horizon and $N$ and $\alpha$ are as defined above. We use $\tau \in\{25,50,100\}$, $N \in\{2,3,4\}$ and $\alpha \in\{1.0,1.2,1.6\}$ so that we get a total of 27 test problems. We note that our test problems are adapted from those in Topaloglu [20].

We use constraint generation to solve $(P L)$. By Lemma 1 we can add constraints of the form $v_{i, t}\left(r_{i}\right)-$ $v_{i, t}\left(r_{i}-1\right) \geq v_{i, t}\left(r_{i}+1\right)-v_{i, t}\left(r_{i}\right)$ for all $t, i$ and $r_{i} \in \mathcal{R}_{i}$ to $(P L)$ without affecting its optimal objective function value. So, while solving $(P L)$, we start with a linear program that only has the above mentioned constraints and the nonnegativity constraints. We add constraints of type (2) on the fly by solving 
the separation problem described in $§ 3.1$. By Proposition 3 and Lemma 3, we can solve the separation problem as a linear program. We add violated constraints to $(P L)$ and stop when we are within $1 \%$ of optimality.

We use constraint generation to solve $(L R)$ as well. It can be verified that there exists an optimal solution $\left\{\hat{\nu}_{i, t}\left(r_{i}\right) \mid \forall t, i, r_{i} \in \mathcal{R}_{i}\right\}$ to $(L R)$ that satisfies $\hat{\nu}_{i, t}\left(r_{i}\right)-\hat{\nu}_{i, t}\left(r_{i}-1\right) \geq \hat{\nu}_{i, t}\left(r_{i}+1\right)-\hat{\nu}_{i, t}\left(r_{i}\right)$ for all $t, i$ and $r_{i} \in \mathcal{R}_{i}$. While solving $(L R)$, we start with a linear program that only has the above mentioned constraints in addition to constraints (5) and the nonnegativity constraints. We add constraints of type (4) on the fly by solving the following separation problem. Given a solution $\left(\left\{\hat{\lambda}_{i, j, t} \mid \forall t, j, i \in\right.\right.$ $\left.\left.\mathcal{I}_{j}\right\},\left\{\hat{\nu}_{i, t}\left(r_{i}\right) \mid \forall t, i, r_{i} \in \mathcal{R}_{i}\right\}\right)$ to the restricted linear program, we check for each $i$ and $t$ if

$$
\max _{r_{i} \in \mathcal{R}_{i}, \boldsymbol{u}^{i} \in \mathcal{U}_{i}\left(r_{i}\right)} \sum_{j \in \mathcal{J}_{i}} p_{j, t} u_{j}^{i}\left[\hat{\lambda}_{i, j, t}+\hat{\nu}_{i, t+1}\left(r_{i}-1\right)-\hat{\nu}_{i, t+1}\left(r_{i}\right)\right]+\hat{\nu}_{i, t+1}\left(r_{i}\right)-\hat{\nu}_{i, t}\left(r_{i}\right)
$$

is greater than zero. Note that the separation problem for $(L R)$ is easy to solve since for $r_{i} \in \mathcal{R}_{i} \backslash\{0\}$, the maximum is attained by setting $u_{j}^{i}=\mathbb{1}_{\left[\hat{\lambda}_{i, j, t}+\hat{\nu}_{i, t+1}\left(r_{i}-1\right)-\hat{\nu}_{i, t+1}\left(r_{i}\right)>0\right]}$ for $j \in \mathcal{J}_{i}$. On the other hand, if $r_{i}=0$, the only feasible solution is $u_{j}^{i}=0$ for all $j \in \mathcal{J}_{i}$. We add violated constraints to $(L R)$ and stop when we are within $1 \%$ of optimality.

\begin{tabular}{ccrcr} 
Problem & \multicolumn{3}{c}{$(P L)$} & \multicolumn{2}{c}{$(L R)$} \\
$(\tau, N, \alpha)$ & $V^{P L}$ & $\mathrm{CPU}$ & $V^{L R}$ & $\mathrm{CPU}$ \\
\hline$(25,2,1.0)$ & 622 & 3 & 622 & 0.1 \\
$(25,2,1.2)$ & 557 & 3 & 557 & 0.1 \\
$(25,2,1.6)$ & 448 & 2 & 448 & 0.1 \\
$(25,3,1.0)$ & 972 & 14 & 972 & 0.4 \\
$(25,3,1.2)$ & 868 & 8 & 868 & 0.3 \\
$(25,3,1.6)$ & 700 & 5 & 700 & 0.2 \\
$(25,4,1.0)$ & 1,187 & 39 & 1,188 & 1 \\
$(25,4,1.2)$ & 1,048 & 21 & 1,048 & 1 \\
$(25,4,1.6)$ & 843 & 10 & 844 & 0.5 \\
\hline$(50,2,1.0)$ & 1,305 & 71 & 1,306 & 1 \\
$(50,2,1.2)$ & 1,117 & 42 & 1,117 & 1 \\
$(50,2,1.6)$ & 908 & 24 & 908 & 0.5 \\
$(50,3,1.0)$ & 2,038 & 496 & 2,038 & 2 \\
$(50,3,1.2)$ & 1,844 & 211 & 1,845 & 2 \\
$(50,3,1.6)$ & 1,500 & 74 & 1,500 & 1 \\
$(50,4,1.0)$ & 2,496 & 1,556 & 2,497 & 6 \\
$(50,4,1.2)$ & 2,260 & 746 & 2,263 & 4 \\
$(50,4,1.6)$ & 1,855 & 227 & 1,856 & 3 \\
\hline$(100,2,1.0)$ & 3,652 & 2,149 & 3,652 & 27 \\
$(100,2,1.2)$ & 3,242 & 1,409 & 3,245 & 18 \\
$(100,2,1.6)$ & 2,599 & 831 & 2,603 & 8 \\
$(100,3,1.0)$ & 5,529 & 17,821 & 5,531 & 44 \\
$(100,3,1.2)$ & 4,967 & 9,314 & 4,972 & 32 \\
$(100,3,1.6)$ & 4,131 & 4,000 & 4,137 & 18 \\
$(100,4,1.0)$ & 6,835 & 108,297 & 6,837 & 80 \\
$(100,4,1.2)$ & 6,141 & 51,708 & 6,148 & 75 \\
$(100,4,1.6)$ & 4,910 & 12,250 & 4,917 & 36 \\
\hline
\end{tabular}

TABLE 1. Comparison of the upper bounds and solution times of $(P L)$ and $(L R)$ both solved to $1 \%$ of optimality by linear programming.

Table 1 gives the objective function values of $(P L)$ and $(L R)$ and the CPU seconds when they are solved to within $1 \%$ of optimality. We solve the test problems using CPLEX 11.2 on a Pentium Core 
2 Duo PC with $3 \mathrm{GHz}$ CPU and 4 GB RAM. The first column gives the characteristics of the test problem in terms of $(\tau, N, \alpha)$. The second column gives the objective function value of $(P L)$, while the third column gives the CPU seconds required by $(P L)$. The fourth and fifth columns do the same thing, but for $(L R)$. Comparing the second and fourth columns, we see that the objective function values of $(P L)$ and $(L R)$ are very close; the differences are within $1 \%$. On the other hand, the solution times for $(P L)$ on the test problems with a relatively large number of spokes and time periods can be orders of magnitude greater than $(L R)$. We note that it is possible to solve both $(P L)$ and $(L R)$ more efficiently; see [20]. Our goal here is to simply compare the objective function values and solution times of comparable implementations of both methods.

6. Conclusions We make the following research contributions in this paper: (1) We show that the approximate dynamic programming approach with piecewise-linear basis functions is solvable in polynomial-time, (2) The resulting compact linear program is equivalent to the linear programming version of the Lagrangian relaxation approach proposed in Topaloglu [20]. This result shows that there might be surprising connections between the approximate dynamic programming approach and the Lagrangian relaxation approach for complicated dynamic programs, and one can benefit from unifying forces as it were. (3) We show that there exists a separable concave approximation that yields the tightest upper bound among all separable piecewise-linear approximations to the value function. This implies that the Lagrangian relaxation approach obtains the tightest upper bound among all separable piecewise-linear approximations that are upper bounds on the value function.

We sketch in the appendix how the polynomial-time solvability extends to network RM with overbooking under some assumptions on the denied-boarding cost function. We show that the result does not directly extend to choice-based network RM and discuss why - our insight is that the number of Lagrange multipliers plays a critical role, requiring problem-specific customization.

As to computational impact, we solved the piecewise-linear approximation using the linearprogramming based separation we describe in this paper, but our results suggest that solving the Lagrangian relaxation, either as a linear program or by subgradient optimization, is still faster. Tong and Topaloglu [19] make a similar observation for the affine relaxation of Adelman [1]; they also find that the Lagrangian relaxation approach described in Kunnumkal and Topaloglu [11] is more efficient. Improving the efficiency of the separation, say by a faster, combinatorial, algorithm, would be an interesting area for future research.

Acknowledgments. We wish to thank the referees and the Associate Editor for many constructive comments that improved the paper. Kalyan Talluri acknowledges research funding from the Ministerio de Economía y Competitividad of Spain under grant ECO2013-41131-R. Sumit Kunnumkal gratefully acknowledges the financial support of the Indian School of Business.

\section{References}

[1] Adelman, D. 2007. Dynamic bid-prices in revenue management. Operations Research 55(4) 647-661.

[2] Adelman, D, A. J. Mersereau. 2008. Relaxations of weakly coupled stochastic dynamic programs. Operations Research 55(3) 712-727.

[3] Bodea, T., M. Ferguson, L. Garrow. 2009. Choice-based revenue management: Data from a major hotel chain. Manufacturing and Service Operations Management 11 356-361.

[4] Bront, J. J. M., I. Méndez-Díaz, G. Vulcano. 2009. A column generation algorithm for choice-based network revenue management. Operations Research 57(3) 769-784.

[5] Erdelyi, A., H. Topaloglu. 2009. A dynamic programming decomposition method for making overbooking decisions over an airline network. INFORMS Journal on Computing .

[6] Gallego, G., G. Iyengar, R. Phillips, A. Dubey. 2004. Managing flexible products on a network. Tech. Rep. TR-2004-01, Dept of Industrial Engineering, Columbia University, NY, NY.

[7] Grötschel, M., L. Lovász, A. Schrijver. 1988. Geometric Algorithms and Combinatorial Optimization, vol. 2. Springer. 
[8] Karaesmen, I., G. J. van Ryzin. 2001. Coordinating overbooking and capacity control decisions on a network. Tech. rep., Graduate School of Business, Columbia University, New York, NY. Working paper.

[9] Kunnumkal, S., K. T. Talluri. 2012. A new compact linear programming formulation for choice network revenue management. Tech. rep., Universitat Pompeu Fabra.

[10] Kunnumkal, S., K. T. Talluri. 2012. Piecewise-linear approximations for choice network revenue management (in preparation). Tech. rep., Universitat Pompeu Fabra.

[11] Kunnumkal, S., H. Topaloglu. 2010. Computing time-dependent bid prices in network revenue management problems. Transportation Science $4438-62$.

[12] Kunnumkal, S., H. Topaloglu. 2010. A new dynamic programming decomposition method for the network revenue management problem with customer choice behavior. Production and Operations Management 19(5) $575-590$.

[13] Liu, Q., G. J. van Ryzin. 2008. On the choice-based linear programming model for network revenue management. Manufacturing and Service Operations Management 10(2) 288-310.

[14] Meissner, J., A. K. Strauss. 2012. Network revenue management with inventory-sensitive bid prices and customer choice. European Journal of Operational Research 216(2) 459-468.

[15] Méndez-Díaz, I., J. Miranda Bront, G. Vulcano, P. Zabala. 2012. A branch-and-cut algorithm for the latentclass logit assortment problem. Discrete Applied Mathematics ((forthcoming)).

[16] Talluri, K. T. 2008. On bounds for network revenue management. Tech. Rep. WP-1066, UPF.

[17] Talluri, K. T., G. J. van Ryzin. 2004. Revenue management under a general discrete choice model of consumer behavior. Management Science 50(1) 15-33.

[18] Talluri, K. T., G. J. van Ryzin. 2004. The Theory and Practice of Revenue Management. Kluwer, New York, NY.

[19] Tong, C., H. Topaloglu. 2014. On approximate linear programming approach for network revenue management problems. INFORMS Journal of Computing 26 131-134.

[20] Topaloglu, H. 2009. Using Lagrangian relaxation to compute capacity-dependent bid prices in network revenue management. Operations Research 57 637-649. 


\section{Appendix}

Proof of Lemma 1: Our analysis is essentially an adaptation of analogous structural results for the revenue management problem on a single resource (Talluri and van Ryzin [18]). We introduce some notation to simplify the expressions. Fixing a resource $l$, we let $\mathcal{R}_{l}\left(r_{l}\right)=\left\{\boldsymbol{x} \in \mathcal{R} \mid x_{l}=r_{l}\right\}$ be the set of capacity vectors where the capacity on resource $l$ is fixed at $r_{l}$. Given a separable piecewise-linear approximation $\mathcal{V}=\left\{v_{i, t}\left(r_{i}\right) \mid \forall t, i, r_{i} \in \mathcal{R}_{i}\right\}$, we let

$$
\begin{gathered}
\epsilon_{l, t}\left(r_{l}, \mathcal{V}\right)=\min _{\boldsymbol{r} \in \mathcal{R}_{l}\left(r_{l}\right), \boldsymbol{u} \in \mathcal{U}(\boldsymbol{r})}\left\{\sum_{i} v_{i, t}\left(r_{i}\right)-\sum_{j} p_{j, t} u_{j}\left[f_{j}+\sum_{i \in \mathcal{I}_{j}}\left[v_{i, t+1}\left(r_{i}-1\right)-v_{i, t+1}\left(r_{i}\right)\right]\right]\right. \\
\left.-\sum_{i} v_{i, t+1}\left(r_{i}\right)\right\}
\end{gathered}
$$

where the argument $\mathcal{V}$ emphasizes the dependence on the given approximation. Note that if $\mathcal{V}$ is feasible to $(P L)$, then $\epsilon_{i, t}\left(r_{i}, \mathcal{V}\right) \geq 0$ for all $t, i$ and $r_{i} \in \mathcal{R}_{i}$. We begin with a preliminary result.

LEMmA 4. There exists an optimal solution $\hat{\mathcal{V}}=\left\{\hat{v}_{i, t}\left(r_{i}\right) \mid \forall t, i, r_{i} \in \mathcal{R}_{i}\right\}$ to $(P L)$ such that for all $t$, $i$ and $r_{i} \in \mathcal{R}_{i}$, we have $\epsilon_{i, t}\left(r_{i}, \hat{\mathcal{V}}\right)=0$.

$L$ et $\mathcal{V}=\left\{v_{i, t}\left(r_{i}\right) \mid \forall t, i, r_{i} \in \mathcal{R}_{i}\right\}$ be an optimal solution to problem $(P L)$. Let $s$ be the largest time index such that there exists a resource $l$ and $r_{l} \in \mathcal{R}_{l}$ with $\epsilon_{l, s}\left(r_{l}, \mathcal{V}\right)>0$. Since $\mathcal{V}$ is feasible, this means that $\epsilon_{i, t}\left(r_{i}, \mathcal{V}\right)=0$ for all $t>s, i$ and $r_{i} \in \mathcal{R}_{i}$. We consider decreasing $v_{l, s}\left(r_{l}\right)$ alone by $\epsilon_{l, s}\left(r_{l}, \mathcal{V}\right)$ leaving all the other elements of $\mathcal{V}$ unchanged. That is, let $\hat{\mathcal{V}}=\left\{\hat{v}_{i, t}\left(r_{i}\right) \mid \forall t, i, r_{i} \in \mathcal{R}_{i}\right\}$ where

$$
\hat{v}_{i, t}(x)= \begin{cases}v_{i, t}(x)-\epsilon_{i, t}(x, v) & \text { if } i=l, t=s, x=r_{l} \\ v_{i, t}(x) & \text { otherwise }\end{cases}
$$

Note that since $\hat{v}_{i, t}\left(r_{i}\right) \leq v_{i, t}\left(r_{i}\right)$ for all $t, i$ and $r_{i} \in \mathcal{R}_{i}$, we have $\sum_{i} \hat{v}_{i, 1}\left(r_{i}^{1}\right) \leq \sum_{i} v_{i, 1}\left(r_{i}^{1}\right)$. Next, we show that $\hat{\mathcal{V}}$ is feasible. Since $\hat{\mathcal{V}}$ differs from $\mathcal{V}$ only in one element, we only have to check those constraints where $\hat{v}_{l, s}\left(r_{l}\right)$ appears. Observe that $\hat{v}_{l, s}\left(r_{l}\right)$ appears only in the constraints for time periods $s-1$ and $s$. For time period $s-1$, we have

$$
\begin{aligned}
& \sum_{j} p_{j, s-1} u_{j}\left[f_{j}+\sum_{i \in \mathcal{I}_{j}} \hat{v}_{i, s}\left(r_{i}-1\right)\right]+\sum_{i}\left[1-\sum_{j \in \mathcal{J}_{i}} p_{j, s-1} u_{j}\right] \hat{v}_{i, s}\left(r_{i}\right) \\
& \quad \leq \sum_{j} p_{j, s-1} u_{j}\left[f_{j}+\sum_{i \in \mathcal{I}_{j}} v_{i, s}\left(r_{i}-1\right)\right]+\sum_{i}\left[1-\sum_{j \in \mathcal{J}_{i}} p_{j, s-1} u_{j}\right] v_{i, s}\left(r_{i}\right) \\
& \leq \sum_{i} v_{i, s-1}\left(r_{i}\right) \\
& =\sum_{i} \hat{v}_{i, s-1}\left(r_{i}\right)
\end{aligned}
$$

for all $\boldsymbol{r} \in \mathcal{R}$ and $\boldsymbol{u} \in \mathcal{U}(\boldsymbol{r})$, where the first inequality follows since $\hat{v}_{i, s}\left(r_{i}\right) \leq v_{i, s}\left(r_{i}\right)$ and $\sum_{j \in \mathcal{J}_{i}} p_{j, s-1} u_{j} \leq$ 1 , the second inequality follows from the feasibility of $\mathcal{V}$ and the equality follows from (20). For time period $s, \hat{v}_{l, s}\left(r_{l}\right)$ appears only in constraints corresponding to $\boldsymbol{r} \in \mathcal{R}_{l}\left(r_{l}\right)$. For $\boldsymbol{r} \in \mathcal{R}_{l}\left(r_{l}\right)$, we have

$$
\begin{aligned}
& \sum_{i} \hat{v}_{i, s}\left(r_{i}\right) \\
& \quad=\sum_{i} v_{i, s}\left(r_{i}\right)-\epsilon_{l, s}\left(r_{l}, \mathcal{V}\right) \\
& \quad \geq \sum_{j} p_{j, s} u_{j}\left[f_{j}+\sum_{i \in \mathcal{I}_{j}}\left\{v_{i, s+1}\left(r_{i}-1\right)-v_{i, s+1}\left(r_{i}\right)\right\}\right]+\sum_{i} v_{i, s+1}\left(r_{i}\right) \\
& =\sum_{j} p_{j, s} u_{j}\left[f_{j}+\sum_{i \in \mathcal{I}_{j}}\left\{\hat{v}_{i, s+1}\left(r_{i}-1\right)-\hat{v}_{i, s+1}\left(r_{i}\right)\right\}\right]+\sum_{i} \hat{v}_{i, s+1}\left(r_{i}\right)
\end{aligned}
$$


for all $\boldsymbol{u} \in \mathcal{U}(\boldsymbol{r})$, where the inequality follows from the definition of $\epsilon_{l, s}\left(r_{l}, \mathcal{V}\right)$ and the last equality follows from (20). Therefore $\hat{\mathcal{V}}$ is feasible, which implies that $\epsilon_{i, t}\left(r_{i}, \hat{\mathcal{V}}\right) \geq 0$ for all $t, i$ and $r_{i} \in \mathcal{R}_{i}$. Next, we note from $(20)$ that $\epsilon_{i, t}\left(r_{i}, \hat{\mathcal{V}}\right)=0$ for all $t>s, i$ and $r_{i} \in \mathcal{R}_{i}$. For time period $s$, since $\hat{v}_{i, s}\left(r_{i}\right) \leq v_{i, s}\left(r_{i}\right)$ and $\hat{v}_{i, s+1}\left(r_{i}\right)=v_{i, s+1}\left(r_{i}\right)$, it follows that $\epsilon_{i, s}\left(r_{i}, \hat{\mathcal{V}}\right) \leq \epsilon_{i, s}\left(r_{i}, \mathcal{V}\right)$. Therefore, if $\epsilon_{i, s}\left(r_{i}, \mathcal{V}\right)$ was zero, then $\epsilon_{i, s}\left(r_{i}, \hat{\mathcal{V}}\right)$ is also zero. Moreover, $\epsilon_{l, s}\left(r_{l}, \hat{\mathcal{V}}\right)=0<\epsilon_{l, s}\left(r_{l}, \mathcal{V}\right)$.

To summarize, $\hat{\mathcal{V}}$ is an optimal solution with $\epsilon_{i, t}\left(r_{i}, \hat{\mathcal{V}}\right)=0$ for all $t>s, i$ and $r_{i} \in \mathcal{R}_{i}$ and $\left|\left\{\epsilon_{i, s}\left(r_{i}, \hat{\mathcal{V}}\right) \mid \epsilon_{i, s}\left(r_{i}, \hat{\mathcal{V}}\right)>0\right\}\right|<\left|\left\{\epsilon_{i, s}\left(r_{i}, \mathcal{V}\right) \mid \epsilon_{i, s}\left(r_{i}, \mathcal{V}\right)>0\right\}\right|$. We repeat the above procedure finitely many times to obtain an optimal solution $\hat{\mathcal{V}}$ with $\epsilon_{i, t}\left(r_{i}, \hat{\mathcal{V}}\right)=0$ for all $t \geq s, i$ and $r_{i} \in \mathcal{R}_{i}$. Repeating the entire procedure for time periods $s-1, \ldots, 1$ completes the proof. Q.E.D.

We are ready to prove Lemma 1 . By Lemma 4, we can pick an optimal solution $\hat{\mathcal{V}}=\left\{\hat{v}_{i, t}\left(r_{i}\right) \mid \forall t, i, r_{i} \in\right.$ $\left.\mathcal{R}_{i}\right\}$ such that $\epsilon_{i, t}\left(r_{i}, \hat{\mathcal{V}}\right)=0$ for all $t, i$ and $r_{i} \in \mathcal{R}_{i}$. The proof proceeds by induction on the time periods. It is easy to see that the result holds for time period $\tau$. Fix a resource $l$ and assume that statements (i) and (ii) of the lemma hold for all time periods $s>t$. We show below that statements (i) and (ii) hold for time period $t$ as well.

Since $\hat{v}_{i, t}(-1)=-\infty$, statement (i) holds trivially for $r_{l}=0$. For $r_{l}=1$, Lemma 4 implies that there exists $\boldsymbol{x} \in \mathcal{R}_{l}(0)$ and $\boldsymbol{u} \in \mathcal{U}(\boldsymbol{x})$ such that

$$
\begin{gathered}
\hat{v}_{l, t}(0)+\sum_{i \neq l} \hat{v}_{i, t}\left(x_{i}\right)=\sum_{\substack{j \notin \mathcal{J}_{l}\\
}} p_{j, t} u_{j}\left[f_{j}+\sum_{i \neq l} \mathbb{1}_{\left[i \in \mathcal{I}_{j}\right]}\left[\hat{v}_{i, t+1}\left(x_{i}-1\right)-\hat{v}_{i, t+1}\left(x_{i}\right)\right]\right] \\
+\hat{v}_{l, t+1}(0)+\sum_{i \neq l} \hat{v}_{i, t+1}\left(x_{i}\right) .
\end{gathered}
$$

where $\mathbb{1}_{[\cdot]}$ denotes the indicator function and we use the fact that since $x_{l}=0, u_{j}=0$ for all $j \in \mathcal{J}_{l}$. Next, consider the capacity vector $\boldsymbol{y}$ with $y_{i}=x_{i}$ for $i \neq l$ and $y_{l}=r_{l}=1$. Since $\boldsymbol{x} \leq \boldsymbol{y}, \mathcal{U}(\boldsymbol{x}) \subset \mathcal{U}(\boldsymbol{y})$ and it follows that $\boldsymbol{u} \in \mathcal{U}(\boldsymbol{y})$. Since $\hat{\mathcal{V}}$ is feasible, we have

$$
\begin{gathered}
\hat{v}_{l, t}(1)+\sum_{i \neq l} \hat{v}_{i, t}\left(x_{i}\right) \geq \sum_{j \notin \mathcal{J}_{l}} p_{j, t} u_{j}\left[f_{j}+\sum_{i \neq l} \mathbb{1}_{\left[i \in \mathcal{I}_{j}\right]}\left[\hat{v}_{i, t+1}\left(x_{i}-1\right)-\hat{v}_{i, t+1}\left(x_{i}\right)\right]\right] \\
+\hat{v}_{l, t+1}(1)+\sum_{i \neq l} \hat{v}_{i, t+1}\left(x_{i}\right) .
\end{gathered}
$$

Subtracting (21) from (22), we have $\hat{v}_{l, t}(1)-\hat{v}_{l, t}(0) \geq \hat{v}_{l, t+1}(1)-\hat{v}_{l, t+1}(0)$.

We next show that statement (i) holds for $r_{l} \in \mathcal{R}_{l} \backslash\{0,1\}$. By Lemma 4 , there exists $\boldsymbol{x} \in \mathcal{R}_{l}\left(r_{l}-1\right)$ and $\boldsymbol{u} \in \mathcal{U}(\boldsymbol{x})$ such that

$$
\begin{aligned}
& \hat{v}_{l, t}\left(r_{l}-1\right)+\sum_{i \neq l} \hat{v}_{i, t}\left(x_{i}\right) \\
& =\sum_{j} p_{j, t} u_{j}\left[f_{j}+\sum_{i \neq l} \mathbb{1}_{\left[i \in \mathcal{I}_{j}\right]}\left[\hat{v}_{i, t+1}\left(x_{i}-1\right)-\hat{v}_{i, t+1}\left(x_{i}\right)\right]+\mathbb{1}_{\left[l \in \mathcal{I}_{j}\right]}\left[\hat{v}_{l, t+1}\left(r_{l}-2\right)-\hat{v}_{l, t+1}\left(r_{l}-1\right)\right]\right] \\
& +\hat{v}_{l, t+1}\left(r_{l}-1\right)+\sum_{i \neq l} \hat{v}_{i, t+1}\left(x_{i}\right) .
\end{aligned}
$$

Now, consider the capacity vector $\boldsymbol{y}$ with $y_{i}=x_{i}$ for $i \neq l$ and $y_{l}=r_{l}$. Since $\boldsymbol{x} \leq \boldsymbol{y}, \mathcal{U}(\boldsymbol{x}) \subset \mathcal{U}(\boldsymbol{y})$ and it follows that $\boldsymbol{u} \in \mathcal{U}(\boldsymbol{y})$. Since $\hat{\mathcal{V}}$ is feasible, we have

$$
\begin{aligned}
& \hat{v}_{l, t}\left(r_{l}\right)+\sum_{i \neq l} \hat{v}_{i, t}\left(x_{i}\right) \\
& \geq \sum_{j} p_{j, t} u_{j}\left[f_{j}+\sum_{i \neq l} \mathbb{1}_{\left[i \in \mathcal{I}_{j}\right]}\left[\hat{v}_{i, t+1}\left(x_{i}-1\right)-\hat{v}_{i, t+1}\left(x_{i}\right)\right]+\mathbb{1}_{\left[l \in \mathcal{I}_{j}\right]}\left[\hat{v}_{l, t+1}\left(r_{l}-1\right)-\hat{v}_{l, t+1}\left(r_{l}\right)\right]\right] \\
& +\hat{v}_{l, t+1}\left(r_{l}\right)+\sum_{i \neq l} \hat{v}_{i, t+1}\left(x_{i}\right) .
\end{aligned}
$$


Subtracting (23) from (24), we get

$$
\begin{aligned}
& \hat{v}_{l, t}\left(r_{l}\right)-\hat{v}_{l, t}\left(r_{l}-1\right) \\
& \quad \geq \sum_{j} p_{j, t} u_{j} \mathbb{1}_{\left[l \in \mathcal{I}_{j}\right]}\left[2 \hat{v}_{l, t+1}\left(r_{l}-1\right)-\hat{v}_{l, t+1}\left(r_{l}\right)-\hat{v}_{l, t+1}\left(r_{l}-2\right)\right]+\hat{v}_{l, t+1}\left(r_{l}\right)-\hat{v}_{l, t+1}\left(r_{l}-1\right) \\
& \quad \geq \hat{v}_{l, t+1}\left(r_{l}\right)-\hat{v}_{l, t+1}\left(r_{l}-1\right) .
\end{aligned}
$$

Note that the last inequality follows, since by induction assumption (ii), we have

$$
2 \hat{v}_{l, t+1}\left(r_{l}-1\right)-\hat{v}_{l, t+1}\left(r_{l}\right)-\hat{v}_{l, t+1}\left(r_{l}-2\right) \geq 0 .
$$

Next, we show that statement (ii) holds for time period $t$. Since $\hat{v}_{i, t}(-1)=-\infty$, statement (ii) holds trivially for $r_{l}=0$. For $r_{l} \in \mathcal{R}_{l} \backslash\left\{0, r_{l}^{1}\right\}$, Lemma 4 implies that there exists $\boldsymbol{x} \in \mathcal{R}_{l}\left(r_{l}+1\right)$ and $\boldsymbol{u} \in \mathcal{U}(\boldsymbol{x})$ such that

$$
\begin{gathered}
\hat{v}_{l, t}\left(r_{l}+1\right)+\sum_{i \neq l} \hat{v}_{i, t}\left(x_{i}\right) \\
=\sum_{j} p_{j, t} u_{j}\left[f_{j}+\sum_{i \neq l} \mathbb{1}_{\left[i \in \mathcal{I}_{j}\right]}\left[\hat{v}_{i, t+1}\left(x_{i}-1\right)-\hat{v}_{i, t+1}\left(x_{i}\right)\right]+\mathbb{1}_{\left[l \in \mathcal{I}_{j}\right]}\left[\hat{v}_{l, t+1}\left(r_{l}\right)-\hat{v}_{l, t+1}\left(r_{l}+1\right)\right]\right] \\
+\hat{v}_{l, t+1}\left(r_{l}+1\right)+\sum_{i \neq l} \hat{v}_{i, t+1}\left(x_{i}\right) .
\end{gathered}
$$

Now consider the capacity vector $\boldsymbol{y}$ with $y_{i}=x_{i}$ for $i \neq l$ and $y_{l}=r_{l}$. Since $r_{l} \geq 1, u_{j} \leq r_{l}$ for all $j \in \mathcal{J}_{l}$. Since $y_{i}=x_{i}$ for $i \neq l$ and $\boldsymbol{u} \in \mathcal{U}(\boldsymbol{x})$, we have that $u_{j} \leq y_{i}$ for all $j \in \mathcal{J}_{i}$. That is, we have $\boldsymbol{u} \in \mathcal{U}(\boldsymbol{y})$. Since $\hat{\mathcal{V}}$ is feasible, we have

$$
\begin{gathered}
\hat{v}_{l, t}\left(r_{l}\right)+\sum_{i \neq l} \hat{v}_{i, t}\left(x_{i}\right) \\
\geq \sum_{j} p_{j, t} u_{j}\left[f_{j}+\sum_{i \neq l} \mathbb{1}_{\left[i \in \mathcal{I}_{j}\right]}\left[\hat{v}_{i, t+1}\left(x_{i}-1\right)-\hat{v}_{i, t+1}\left(x_{i}\right)\right]+\mathbb{1}_{\left[l \in \mathcal{I}_{j}\right]}\left[\hat{v}_{l, t+1}\left(r_{l}-1\right)-\hat{v}_{l, t+1}\left(r_{l}\right)\right]\right] \\
+\hat{v}_{l, t+1}\left(r_{l}\right)+\sum_{i \neq l} \hat{v}_{i, t+1}\left(x_{i}\right) .
\end{gathered}
$$

Subtracting (27) from (26), we get

$$
\begin{aligned}
\hat{v}_{l, t} & \left(r_{l}+1\right)-\hat{v}_{l, t}\left(r_{l}\right) \\
& \leq \sum_{j} p_{j, t} u_{j} \mathbb{1}_{\left[l \in \mathcal{I}_{j}\right]}\left[2 \hat{v}_{l, t+1}\left(r_{l}\right)-\hat{v}_{l, t+1}\left(r_{l}+1\right)-\hat{v}_{l, t+1}\left(r_{l}-1\right)\right]+\hat{v}_{l, t+1}\left(r_{l}+1\right)-\hat{v}_{l, t+1}\left(r_{l}\right) \\
& \leq 2 \hat{v}_{l, t+1}\left(r_{l}\right)-\hat{v}_{l, t+1}\left(r_{l}+1\right)-\hat{v}_{l, t+1}\left(r_{l}-1\right)+\hat{v}_{l, t+1}\left(r_{l}+1\right)-\hat{v}_{l, t+1}\left(r_{l}\right) \\
& =\hat{v}_{l, t+1}\left(r_{l}\right)-\hat{v}_{l, t+1}\left(r_{l}-1\right) \\
& \leq \hat{v}_{l t}\left(r_{l}\right)-\hat{v}_{l t}\left(r_{l}-1\right) .
\end{aligned}
$$

Note that the second inequality above follows, since by induction assumption (ii), $2 \hat{v}_{l, t+1}\left(r_{l}\right)-\hat{v}_{l, t+1}\left(r_{l}+\right.$ $1)-\hat{v}_{l, t+1}\left(r_{l}-1\right) \geq 0$ and $\sum_{j} p_{j, t} u_{j} \mathbb{1}_{\left[l \in \mathcal{I}_{j}\right]} \leq 1$ and the last inequality follows from (25). Finally, for $r_{l}=r_{l}^{1}$, following a similar analysis, we get

$$
\begin{gathered}
\hat{v}_{l, t}\left(r_{l}^{1}\right)-\hat{v}_{l, t}\left(r_{l}^{1}-1\right) \geq \sum_{j} p_{j, t} u_{j} \mathbb{1}_{\left[l \in \mathcal{I}_{j}\right]}\left[2 \hat{v}_{l, t+1}\left(r_{l}^{1}-1\right)-\hat{v}_{l, t+1}\left(r_{l}^{1}\right)-\hat{v}_{l, t+1}\left(r_{l}^{1}-2\right)\right] \\
+\hat{v}_{l, t+1}\left(r_{l}^{1}\right)-\hat{v}_{l, t+1}\left(r_{l}^{1}-1\right) .
\end{gathered}
$$

By induction assumption (ii), $2 \hat{v}_{l, t+1}\left(r_{l}^{1}-1\right)-\hat{v}_{l, t+1}\left(r_{l}^{1}\right)-\hat{v}_{l, t+1}\left(r_{l}^{1}-2\right) \geq 0$ and $\hat{v}_{l, t+1}\left(r_{l}^{1}\right)-\hat{v}_{l, t+1}\left(r_{l}^{1}-1\right) \geq$ $\hat{v}_{l, t+1}\left(r_{l}^{1}+1\right)-\hat{v}_{l, t+1}\left(r_{l}^{1}\right)$. We have

$$
\hat{v}_{l, t}\left(r_{l}^{1}\right)-\hat{v}_{l, t}\left(r_{l}^{1}-1\right) \geq \hat{v}_{l, t+1}\left(r_{l}^{1}+1\right)-\hat{v}_{l, t+1}\left(r_{l}^{1}\right)=0=\hat{v}_{l, t}\left(r_{l}^{1}+1\right)-\hat{v}_{l, t}\left(r_{l}^{1}\right) .
$$

Therefore, statements (i) and (ii) hold at time period $t$ for resource $l$. This completes the proof since resource $l$ was an arbitrary choice. Q.E.D. 
Proof of Proposition 1: Suppose for product $j$ and time $t$, we have $\sum_{i^{\prime} \in \mathcal{I}_{j}} \lambda_{i^{\prime}, j, t}>f_{j}$. This implies that for some $i \in \mathcal{I}_{j}, \lambda_{i, j, t}>0$. Let $\delta=\min \left\{\lambda_{i, j, t}, \sum_{i^{\prime} \in \mathcal{I}_{j}} \lambda_{i^{\prime}, j, t}-f_{j}\right\}>0$ and let $\left\{\hat{\lambda}_{i^{\prime}, j^{\prime}, t^{\prime}} \mid \forall t^{\prime}, j^{\prime}, i^{\prime} \in \mathcal{I}_{j^{\prime}}\right\}$ be the same as $\left\{\lambda_{i^{\prime}, j^{\prime}, t^{\prime}} \mid \forall t^{\prime}, j^{\prime}, i^{\prime} \in \mathcal{I}_{j^{\prime}}\right\}$ except that $\hat{\lambda}_{i, j, t}=\lambda_{i, j, t}-\delta<\lambda_{i, j, t}$.

Note that $\vartheta_{i, 1}^{\hat{\lambda}}\left(r_{i}^{1}\right) \leq \vartheta_{i, 1}^{\lambda}\left(r_{i}^{1}\right)$ as we have reduced the revenue associated with product $j$ at time period $t$, keeping all other product revenues the same. As the first part of the right hand side of (3) is unaffected by this change, we get

$$
V_{1}^{\hat{\lambda}}\left(\boldsymbol{r}^{1}\right) \leq V_{1}^{\lambda}\left(\boldsymbol{r}^{1}\right) .
$$

If after performing this step, we still have $\sum_{i^{\prime} \in \mathcal{I}_{j}} \hat{\lambda}_{i^{\prime}, j, t}>f_{j}$ for some product $j$ and time $t$, we can repeat this step for another resource $i \in \mathcal{I}_{j}$ until we have $\sum_{i^{\prime} \in \mathcal{I}_{j}} \lambda_{i^{\prime}, j, t} \leq f_{j}$ for all $j$ and $t$.

Now suppose for some product $j$ and time $t, \sum_{i^{\prime} \in \mathcal{I}_{j}} \lambda_{i^{\prime}, j, t}<f_{j}$. Fix $i \in \mathcal{I}_{j}$ and let $\left\{\hat{\lambda}_{i^{\prime}, j^{\prime}, t^{\prime}} \mid \forall t^{\prime}, j^{\prime}, i^{\prime} \in\right.$ $\left.\mathcal{I}_{j^{\prime}}\right\}$ be the same as $\left\{\lambda_{i^{\prime}, j^{\prime}, t^{\prime}} \mid \forall t^{\prime}, j^{\prime}, i^{\prime} \in \mathcal{I}_{j^{\prime}}\right\}$ except that $\hat{\lambda}_{i, j, t}=\lambda_{i, j, t}+\delta$, where $\delta=f_{j}-\sum_{i \in \mathcal{I}_{j}} \lambda_{i, j, t}>0$.

We have

$$
\vartheta_{i, 1}^{\hat{\lambda}}\left(r_{i}^{1}\right) \leq \vartheta_{i, 1}^{\lambda}\left(r_{i}^{1}\right)+\delta
$$

This is because, by increasing the revenue associated with product $j$ at time $t$ by $\delta$, while keeping all other product revenues the same, we cannot increase the optimal expected revenue from resource $i$ by more than $\delta$. However,

$$
\left[f_{j}-\sum_{i^{\prime} \in \mathcal{I}_{j}} \hat{\lambda}_{i^{\prime}, j, t}\right]^{+}=\left[f_{j}-\sum_{i^{\prime} \in \mathcal{I}_{j}} \lambda_{i^{\prime}, j, t}\right]^{+}-\delta
$$

and so

$$
V_{1}^{\hat{\lambda}}\left(\boldsymbol{r}^{1}\right) \leq V_{1}^{\lambda}\left(\boldsymbol{r}^{1}\right) .
$$

By repeating this step, if necessary, we obtain an optimal solution that satisfies $\sum_{i^{\prime} \in \mathcal{I}_{j}} \lambda_{i^{\prime}, j, t}=f_{j}$ for all $j$ and $t$.

Finally, we show that there exists optimal Lagrange multipliers $\lambda_{i^{\prime}, j^{\prime}, t^{\prime}} \geq 0$ for all $t^{\prime}, j^{\prime}$ and $i^{\prime} \in \mathcal{I}_{j^{\prime}}$. Suppose $\lambda_{i, j, t}<0$ for some product $j$, resource $i \in \mathcal{I}_{j}$ and time $t$. In this case, observe that $u_{j}^{i t}=0$. That is, we reject product $j$ at time $t$ on resource $i$. Since the Lagrange multipliers sum up to $f_{j}$, there exists a resource $l \in \mathcal{I}_{j}$ such that $\lambda_{l, j, t}>0$. Let $\delta=\min \left\{\left|\lambda_{i, j, t}\right|, \lambda_{l, j, t}\right\}>0$ and let $\left\{\hat{\lambda}_{i^{\prime}, j^{\prime}, t^{\prime}} \mid \forall t^{\prime}, j^{\prime}, i^{\prime} \in \mathcal{I}_{j^{\prime}}\right\}$ be the same as $\left\{\lambda_{i^{\prime}, j^{\prime}, t^{\prime}} \mid \forall t^{\prime}, j^{\prime}, i^{\prime} \in \mathcal{I}_{j^{\prime}}\right\}$ except that $\hat{\lambda}_{i, j, t}=\lambda_{i, j, t}+\delta$ and $\hat{\lambda}_{l, j, t}=\lambda_{l, j, t}-\delta$. Observe that $\hat{\lambda}_{i, j, t} \leq 0$ and we continue to reject product $j$ at time $t$ on resource $i$. Therefore $\vartheta_{i, 1}^{\lambda}\left(r_{i}^{1}\right)=\vartheta_{i, 1}^{\lambda}\left(r_{i}^{1}\right)$. On the other hand, $\vartheta_{l, 1}^{\hat{\lambda}}\left(r_{l}^{1}\right) \leq \vartheta_{l, 1}^{\lambda}\left(r_{l}^{1}\right)$ so that $V_{1}^{\hat{\lambda}}\left(\boldsymbol{r}^{1}\right) \leq V_{1}^{\lambda}\left(\boldsymbol{r}^{1}\right)$. If there is still some Lagrange multiplier that is negative, we repeat the step until we have $\lambda_{i^{\prime}, j^{\prime}, t^{\prime}} \geq 0$ for all $t^{\prime}, j^{\prime}$ and $i^{\prime} \in \mathcal{I}_{j^{\prime}}$. Q.E.D.

Proof of Lemma 3: Note that an optimal solution to problem (7) satisfies $u_{j}^{i}=\mathbb{1}_{\left[\lambda_{i, j, t} \geq \psi_{i, t+1}\left(r_{i}\right)\right]}$ for all $j \in \mathcal{J}_{i}$, where we use the fact that $\psi_{i, t+1}(0)=\infty$. Therefore, using the convention that $0 \times-\infty=0$, problem (7) can be written as $\max _{r \in \mathcal{R}_{i}} \sum_{j \in \mathcal{J}_{i}} p_{j, t} \mathbb{1}_{\left[\lambda_{i, j, t} \geq \psi_{i, t+1}(r)\right]}\left[\lambda_{i, j, t}-\psi_{i, t+1}(r)\right]+\Delta_{i, t}(r)$. On the other hand, there exists an optimal solution $\left(\hat{w}_{i, t},\left\{\hat{z}_{i, j, t, r} \mid \forall j \in \mathcal{J}_{i}, r \in \mathcal{R}_{i}\right\}\right)$ to $\left(\operatorname{SepLR} R_{i}\right)$ such that $\hat{z}_{i, j, t, r}=$ $p_{j, t}\left[\lambda_{i, j, t}-\psi_{i, t+1}(r)\right]^{+}=p_{j, t} \mathbb{1}_{\left[\lambda_{i, j, t} \geq \psi_{i, t+1}(r)\right]}\left[\lambda_{i, j, t}-\psi_{i, t+1}(r)\right]$ for all $j \in \mathcal{J}_{i}$ and $r \in \mathcal{R}_{i}$. Moreover, there exists an $\hat{r} \in \mathcal{R}_{i}$ such that $\hat{w}_{i, t}=\sum_{j \in \mathcal{J}_{i}} \hat{z}_{i, j, t, \hat{r}}+\Delta_{i, t}(\hat{r})$. Therefore, $\hat{w}_{i, t}=\max _{r \in \mathcal{R}_{i}} \sum_{j \in \mathcal{J}_{i}} \hat{z}_{i, j, t, r}+\Delta_{i, t}(r)$. Q.E.D.

Proof of Proposition 4: Since we focus on the separation problem at time $t$, in the following, we drop the time subscript and write $B_{i, t}(\hat{\lambda}, \hat{w}, \hat{z})$ as $B_{i}(\hat{\lambda}, \hat{w}, \hat{z})$ to reduce the notational burden. Choose $(\hat{\lambda}, \hat{w}, \hat{z})$ to be an optimal solution to $(\operatorname{SepLR})$ with a minimal set $\bigcup_{i^{\prime} \in \mathcal{I}} B_{i^{\prime}}(\hat{\lambda}, \hat{w}, \hat{z})$. So, there is no other optimal solution $\left(\hat{\lambda}^{\prime}, \hat{w}^{\prime}, \hat{z}^{\prime}\right)$ which has a set of binding constraints that is a strict subset of the binding constraints of $(\hat{\lambda}, \hat{w}, \hat{z})$; that is,

$$
\bigcup_{i^{\prime} \in \mathcal{I}} B_{i^{\prime}}\left(\hat{\lambda}^{\prime}, \hat{w}^{\prime}, \hat{z}^{\prime}\right) \subsetneq \bigcup_{i^{\prime} \in \mathcal{I}} B_{i^{\prime}}(\hat{\lambda}, \hat{w}, \hat{z}) .
$$


For resource $i^{\prime}$, we let $\hat{r}_{i^{\prime}}=\max \left\{r \mid r \in B_{i^{\prime}}(\hat{\lambda}, \hat{w}, \hat{z})\right\}$, so that for all $r>\hat{r}_{i^{\prime}}, \xi_{i^{\prime}, t}(r)>0$. Now suppose there exists a product $j$ such that for $i \in \mathcal{I}_{j}$ we have $\hat{\lambda}_{i, j, t}<\psi_{i, t+1}\left(\hat{r}_{i}\right)$, while for $l \in \mathcal{I}_{j}$, we have $\hat{\lambda}_{l, j, t}>\psi_{l, t+1}\left(\hat{r}_{l}\right)$. In this case, we construct an optimal solution $(\bar{\lambda}, \bar{w}, \bar{z})$ with $\bigcup_{i^{\prime} \in \mathcal{I}} B_{i^{\prime}}(\bar{\lambda}, \bar{w}, \bar{z}) \subsetneq \bigcup_{i^{\prime} \in \mathcal{I}} B_{i^{\prime}}(\hat{\lambda}, \hat{w}, \hat{z})$, which contradicts $(\hat{\lambda}, \hat{w}, \hat{z})$ being an optimal solution with a minimal set of binding constraints of type (14) amongst all optimal solutions.

Recall (from Lemma 1) that $\bar{v}_{i^{\prime}, t}\left(r_{i^{\prime}}\right)-\bar{v}_{i^{\prime}, t}\left(r_{i^{\prime}}-1\right) \geq \bar{v}_{i^{\prime}, t}\left(r_{i^{\prime}}+1\right)-\bar{v}_{i^{\prime}, t}\left(r_{i^{\prime}}\right)$ for all $i^{\prime}, r_{i^{\prime}} \in \mathcal{R}_{i^{\prime}}$. Thus, we have

$$
\psi_{i^{\prime}, t+1}\left(r_{i^{\prime}}\right) \geq \psi_{i^{\prime}, t+1}\left(r_{i^{\prime}}+1\right) \geq 0, \quad \forall r_{i^{\prime}} \in \mathcal{R}_{i^{\prime}}
$$

( $\geq 0$ as we set $\bar{v}_{i^{\prime}, t}\left(r_{i^{\prime}}^{1}+1\right)=\bar{v}_{i^{\prime}, t}\left(r_{i^{\prime}}^{1}\right)$; cf. Lemma 1$)$. Also recall that we had assumed that $p_{j, t}>0$ without loss of generality.

Let

$$
\epsilon=\min \left\{\psi_{i, t+1}\left(\hat{r}_{i}\right)-\hat{\lambda}_{i, j, t}, \hat{\lambda}_{l, j, t}-\psi_{l, t+1}\left(\hat{r}_{l}\right), \min \left\{\xi_{i, t}(r) \mid r \in B_{i}^{c}(\hat{\lambda}, \hat{w}, \hat{z})\right\}\right\}>0,
$$

with the understanding that if $B_{i}^{c}(\hat{\lambda}, \hat{w}, \hat{z})$ is empty, then $\min \left\{\xi_{i, t}(r) \mid r \in B_{i}^{c}(\hat{\lambda}, \hat{w}, \hat{z})\right\}=\infty$.

We construct a solution $(\bar{\lambda}, \bar{w}, \bar{z})$ in the following manner. Pick $\delta \in(0, \epsilon)$ and let

$$
\bar{\lambda}_{i^{\prime}, k, t}= \begin{cases}\hat{\lambda}_{i, j, t}+\delta & \text { if } i^{\prime}=i, k=j \\ \hat{\lambda}_{l, j, t}-\delta & \text { if } i^{\prime}=l, k=j \\ \hat{\lambda}_{i^{\prime}, k, t} & \text { otherwise }\end{cases}
$$

$\bar{w}_{i^{\prime}, t}=\hat{w}_{i^{\prime}, t}$ for all $i^{\prime}$ and

$$
\bar{z}_{i^{\prime}, k, t, r}= \begin{cases}p_{j, t}\left[\bar{\lambda}_{i, j, t}-\psi_{i, t+1}(r)\right]^{+} & \text {if } i^{\prime}=i, k=j, r \in B_{i}^{c} \\ p_{j, t}\left[\bar{\lambda}_{l, j, t}-\psi_{l, t+1}(r)\right]^{+} & \text {if } i^{\prime}=l, k=j, r \in \mathcal{R}_{l} \\ \hat{z}_{i^{\prime}, k, t, r} & \text { otherwise. }\end{cases}
$$

Note that by construction, $(\bar{\lambda}, \bar{w}, \bar{z})$ has the same elements as $(\hat{\lambda}, \hat{w}, \hat{z})$ except that $\bar{\lambda}_{i, j, t}=\hat{\lambda}_{i, j, t}+\delta$, $\bar{\lambda}_{l, j, t}=\hat{\lambda}_{l, j, t}-\delta, \bar{z}_{i, j, t, r}=p_{j, t}\left[\bar{\lambda}_{i, j, t}-\psi_{i, t+1}(r)\right]^{+}$for $r \in B_{i}^{c}(\hat{\lambda}, \hat{w}, \hat{z})$ and $\bar{z}_{l, j, t, r}=p_{j, t}\left[\bar{\lambda}_{l, j, t}-\psi_{l, t+1}(r)\right]^{+}$for all $r \in \mathcal{R}_{l}$. We begin with some preliminary results.

Lemma 5. (i) The solution $(\bar{\lambda}, \bar{w}, \bar{z})$ satisfies constraints (14), (15) and (18) for all $i^{\prime} \notin\{i, l\}$.

(ii) $B_{i^{\prime}}(\bar{\lambda}, \bar{w}, \bar{z})=B_{i^{\prime}}(\hat{\lambda}, \hat{w}, \hat{z})$ for all $i^{\prime} \notin\{i, l\}$.

$T$ he proof follows by noting that $(\hat{\lambda}, \hat{w}, \hat{z})$ is feasible and $\bar{w}_{i^{\prime}, t}=\hat{w}_{i^{\prime}, t}, \bar{\lambda}_{i^{\prime}, k, t}=\hat{\lambda}_{i^{\prime}, k, t}$ and $\bar{z}_{i^{\prime}, k, t, r}=$ $\hat{z}_{i^{\prime}, k, t, r}$ for all $i^{\prime} \notin\{i, l\}, r \in \mathcal{R}_{i^{\prime}}$ and $k \in \mathcal{J}_{i^{\prime}}$. Q.E.D.

Lemma 6. (i) The solution $(\bar{\lambda}, \bar{w}, \bar{z})$ satisfies constraints (14), (15) and (18) for resource $i$.

(ii) $B_{i}(\bar{\lambda}, \bar{w}, \bar{z})=B_{i}(\hat{\lambda}, \hat{w}, \hat{z})$.

$W$ e first consider constraints (14). For $r \in B_{i}^{c}(\hat{\lambda}, \hat{w}, \hat{z})$, we have

$$
\bar{z}_{i, j, t, r}=p_{j, t}\left[\hat{\lambda}_{i, j, t}+\delta-\psi_{i, t+1}(r)\right]^{+} \leq p_{j, t}\left[\hat{\lambda}_{i, j, t}-\psi_{i, t+1}(r)\right]^{+}+p_{j, t} \delta<\hat{z}_{i, j, t, r}+\epsilon \leq \hat{z}_{i, j, t, r}+\xi_{i, t}(r),
$$

where the second inequality uses $\delta<\epsilon$, and the fact that since $(\hat{\lambda}, \hat{w}, \hat{z})$ is feasible, $\hat{z}_{i, j, t, r} \geq p_{j, t}\left[\hat{\lambda}_{i, j, t}-\right.$ $\left.\psi_{i, t+1}(r)\right]$ and $\hat{z}_{i, j, t, r} \geq 0$. Therefore, for all $r \in B_{i}^{c}(\hat{\lambda}, \hat{w}, \hat{z}), \bar{w}_{i, t}=\hat{w}_{i, t}=\sum_{k \in \mathcal{J}_{i}, k \neq j} \hat{z}_{i, k, t, r}+\hat{z}_{i, j, t, r}+$ $\Delta_{i, t}(r)+\xi_{i, t}(r)>\sum_{k \in \mathcal{J}_{i}} \bar{z}_{i, k, t, r}+\Delta_{i, t}(r)$, where the second equality follows by definition of $\xi_{i, t}(r)$ and the inequality uses (30) and the fact that $\bar{z}_{i, k, t, r}=\hat{z}_{i, k, t, r}$ for all $k \neq j$. Note also that the constraint is nonbinding for $(\bar{\lambda}, \bar{w}, \bar{z})$. Since $r \in B_{i}^{c}(\hat{\lambda}, \hat{w}, \hat{z})$, the constraint was nonbinding for $(\hat{\lambda}, \hat{w}, \hat{z})$ as well. On the other hand, for $r \in B_{i}(\hat{\lambda}, \hat{w}, \hat{z})$ since we have $\bar{z}_{i, j, t, r}=\hat{z}_{i, j, t, r}$ and $\bar{w}_{i, t}=\hat{w}_{i, t},(\bar{\lambda}, \bar{w}, \bar{z})$ satisfies constraint (14) as an equality. Therefore, constraints (14) are binding for $(\bar{\lambda}, \bar{w}, \bar{z})$. Since $r \in B_{i}(\hat{\lambda}, \hat{w}, \hat{z})$, these constraints were binding for $(\hat{\lambda}, \hat{w}, \hat{z})$ as well. Therefore, $B_{i}(\bar{\lambda}, \bar{w}, \bar{z})=B_{i}(\hat{\lambda}, \hat{w}, \hat{z})$, which proves part (ii) of the lemma.

To complete the proof of part(i), we verify that constraints (15) and (18) are satisfied by $(\bar{\lambda}, \bar{w}, \bar{z})$. For $r \in B_{i}^{c}(\hat{\lambda}, \hat{w}, \hat{z}), \bar{z}_{i, j, t, r}=p_{j, t}\left[\bar{\lambda}_{i, j, t}-\psi_{i, t+1}(r)\right]^{+}$satisfies constraints (15) and (18). For $r \in B_{i}(\hat{\lambda}, \hat{w}, \hat{z})$, 
since $\hat{r}_{i}=\max \left\{r \mid r \in B_{i}(\hat{\lambda}, \hat{w}, \hat{z})\right\}$, we have $\psi_{i, t+1}(r) \geq \psi_{i, t+1}\left(\hat{r}_{i}\right)$ (from 28). Constraint (15) is satisfied since

$$
\begin{array}{r}
p_{j, t}\left[\bar{\lambda}_{i, j, t}-\psi_{i, t+1}(r)\right]=p_{j, t}\left[\hat{\lambda}_{i, j, t}+\delta-\psi_{i, t+1}(r)\right] \leq p_{j, t}\left[\hat{\lambda}_{i, j, t}+\delta-\psi_{i, t+1}\left(\hat{r}_{i}\right)\right]<p_{j, t}\left[\begin{array}{l}
\left.\hat{\lambda}_{i, j, t}+\epsilon-\psi_{i, t+1}\left(\hat{r}_{i}\right)\right] \\
\leq 0 \leq \hat{z}_{i, j, t, r}=\bar{z}_{i, j, t, r},
\end{array}\right.
\end{array}
$$

where the penultimate inequality follows from (29). Constraint (18) is satisfied since for $r \in B_{i}(\hat{\lambda}, \hat{w}, \hat{z})$, $\bar{z}_{i, j, t, r}=\hat{z}_{i, j, t, r} \geq 0$. Q.E.D.

LEMma 7. (i) The solution $(\bar{\lambda}, \bar{w}, \bar{z})$ satisfies constraints (14), (15) and (18) for resource $l$. (ii) $B_{l}(\bar{\lambda}, \bar{w}, \bar{z}) \subsetneq B_{l}(\hat{\lambda}, \hat{w}, \hat{z})$.

$W$ e first consider constraints (14). By definition, $\bar{z}_{l, j, t, r}=p_{j, t}\left[\bar{\lambda}_{l, j, t}-\psi_{l, t+1}(r)\right]^{+}$for all $r \in \mathcal{R}_{l}$. On the other hand, since $(\hat{\lambda}, \hat{w}, \hat{z})$ is a feasible solution, $\hat{z}_{l, j, t, r} \geq p_{j, t}\left[\hat{\lambda}_{l, j, t}-\psi_{i, t+1}(r)\right]^{+}$for all $r \in \mathcal{R}_{l}$. Since $\hat{\lambda}_{l, j, t}>\bar{\lambda}_{l, j, t}$, we have $\hat{z}_{l, j, t, r} \geq \bar{z}_{l, j, t, r}$ for all $r \in \mathcal{R}_{l}$. Using the fact that $\bar{z}_{l, k, t, r}=\hat{z}_{l, k, t, r}$ for all $k \neq j$ and $r \in \mathcal{R}_{l}$, we have

$$
\bar{w}_{l, t}=\hat{w}_{l, t} \geq \sum_{k \in \mathcal{I}_{l}} \hat{z}_{l, k, t, r}+\Delta_{l, t}(r)=\sum_{k \in \mathcal{I}_{l}} \bar{z}_{l, k, t, r}+\Delta_{l, t}(r)
$$

for all $r \in \mathcal{R}_{l}$. Therefore $(\bar{\lambda}, \bar{w}, \bar{z})$ satisfies constraints (14).

Next, we look at the number of binding constraints of type (14). Since $\bar{w}_{l, t}=\hat{w}_{l, t}$ and $\bar{z}_{l, k, t, r} \leq \hat{z}_{l, k, t, r}$ for all $k$ and $r \in \mathcal{R}_{l}, B_{l}(\bar{\lambda}, \bar{w}, \bar{z}) \subseteq B_{l}(\hat{\lambda}, \hat{w}, \hat{z})$. By definition $\hat{r}_{l} \in B_{l}(\hat{\lambda}, \hat{w}, \hat{z})$. We show that $\hat{r}_{l} \notin B_{l}(\bar{\lambda}, \bar{w}, \bar{z})$, which proves part (ii) of the lemma. First, note that

$$
\bar{\lambda}_{l, j, t}-\psi_{l, t+1}\left(\hat{r}_{l}\right)=\hat{\lambda}_{l, j, t}-\delta-\psi_{l, t+1}\left(\hat{r}_{l}\right)>\hat{\lambda}_{l, j, t}-\epsilon-\psi_{l, t+1}\left(\hat{r}_{l}\right) \geq 0
$$

where the last inequality uses (29). Therefore,

$$
\begin{array}{r}
\bar{z}_{l, j, t, \hat{r}_{l}}=p_{j, t}\left[\bar{\lambda}_{l, j, t}-\psi_{l, t+1}\left(\hat{r}_{l}\right)\right]^{+}=p_{j, t}\left[\bar{\lambda}_{l, j, t}-\psi_{l, t+1}\left(\hat{r}_{l}\right)\right]<p_{j, t}\left[\hat{\lambda}_{l, j, t}-\psi_{l, t+1}\left(\hat{r}_{l}\right)\right] \\
\leq p_{j, t}\left[\hat{\lambda}_{l, j, t}-\psi_{l, t+1}\left(\hat{r}_{l}\right)\right]^{+} \leq \hat{z}_{l, j, t, \hat{r}_{l}}
\end{array}
$$

where the second equality follows from (31), the first inequality holds since $\bar{\lambda}_{l, j, t}<\hat{\lambda}_{l, j, t}$ and the last inequality holds since $(\hat{\lambda}, \hat{w}, \hat{z})$ is a feasible solution. The above chain of inequalities imply that $\bar{z}_{l, j, t, \hat{r}_{l}}<$ $\hat{z}_{l, j, t, \hat{r}_{l}}$. Therefore, we have

$$
\bar{w}_{l, t}=\hat{w}_{l, t}=\sum_{k \in \mathcal{J}_{l}, k \neq j} \hat{z}_{l, k, t, \hat{r}_{l}}+\hat{z}_{l, j, t, \hat{r}_{l}}+\Delta_{l, t}\left(\hat{r}_{l}\right)>\sum_{k \in \mathcal{J}_{l}, k \neq j} \bar{z}_{l, k, t, \hat{r}_{l}}+\bar{z}_{l, j, t, \hat{r}_{l}}+\Delta_{l, t}\left(\hat{r}_{l}\right)
$$

Consequently, $\hat{r}_{l} \notin B_{l}(\bar{\lambda}, \bar{w}, \bar{z})$ and $B_{l}(\bar{\lambda}, \bar{w}, \bar{z}) \subsetneq B_{l}(\hat{\lambda}, \hat{w}, \hat{z})$.

To complete the proof of part(i), we verify that constraints (15) and (18) are satisfied by $(\bar{\lambda}, \bar{w}, \bar{z})$. This is trivial, since by definition we have $\bar{z}_{l, j, t, r}=p_{j, t}\left[\bar{\lambda}_{l, j, t}-\psi_{l, t+1}(r)\right]^{+}$. Q.E.D.

We are ready to prove Proposition 4 . By construction $(\bar{\lambda}, \bar{w}, \bar{z})$ satisfies constraints (16). Since $\bar{\lambda}_{l, j, t}>$ $\hat{\lambda}_{l, j, t}-\epsilon \geq \psi_{l, t+1}\left(\hat{r}_{l}\right) \geq 0$ (from 28), $(\bar{\lambda}, \bar{w}, \bar{z})$ also satisfies constraints (17). This together with parts (i) of Lemmas 5, 6 and 7 imply that $(\bar{\lambda}, \bar{w}, \bar{z})$ is a feasible solution. Since $\bar{w}_{i^{\prime}, t}=\hat{w}_{i^{\prime}, t}$ for all $i^{\prime},(\bar{\lambda}, \bar{w}, \bar{z})$ is also optimal. Parts (ii) of Lemmas 5, 6 and 7 together imply that

$$
\bigcup_{i^{\prime} \in \mathcal{I}} B_{i^{\prime}}(\bar{\lambda}, \bar{w}, \bar{z}) \subsetneq \bigcup_{i^{\prime} \in \mathcal{I}} B_{i^{\prime}}(\hat{\lambda}, \hat{w}, \hat{z})
$$

That is, $(\bar{\lambda}, \bar{w}, \bar{z})$ is an optimal solution whose set of binding constraints is a strict subset of those of $(\hat{\lambda}, \hat{w}, \hat{z})$, and we get a contradiction to our minimality assumption. Q.E.D. 
Comparing the Lagrangian relaxation bound with the piecewise-linear bound for network RM with customer choice The following example, drawn from Kunnumkal and Talluri [10], illustrates that the Lagrangian bound can be weaker than the piecewise-linear approximation bound for choice network RM. Consider a network RM problem with two products, two resources and a single time period in the booking horizon. The first product uses only the first resource, while the second product uses only the second resource, and we have a single unit of capacity on each resource. Note that in the airline context, this example corresponds to a parallel flights network. The revenues associated with the products are $f_{1}=10$ and $f_{2}=1$. The choice probabilities are given in Table 2 . In the Lagrangian relaxation, since we have Lagrange multipliers only for $j \in \mathcal{J}_{i}$, we have only two multipliers $\lambda_{1,1,1}$ and $\lambda_{2,2,1}$. Moreover, the constraint $\sum_{i \in \mathcal{I}_{j}} \lambda_{i, j, t}=f_{j}$ implies that all feasible Lagrange multipliers satisfy $\lambda_{1,1,1}=f_{1}$ and $\lambda_{2,2,1}=f_{2}$. Noting that there is only a single time period in the booking horizon and that $\mathcal{Q}_{i}(1)=\mathcal{J}$ for $i=1,2$,

$$
\vartheta_{11}^{\lambda}(1)=\max _{S \subset \mathcal{J}} p_{1}(S) f_{1}=5
$$

and

$$
\vartheta_{21}^{\lambda}(1)=\max _{S \subset \mathcal{J}} p_{2}(S) f_{2}=10 / 11
$$

so that $V^{C L R}=65 / 11$. We note that the offer set on the first resource is $\{1\}$, while the offer set on the second resource is $\{2\}$. Thus, the Lagrange multipliers are not able to coordinate the offer sets across the two resources.

Letting $S_{1}=\{1\}, S_{2}=\{2\}$ and $S_{3}=\{1,2\}$, the linear program associated with the piecewise-linear approximation is

$$
\begin{aligned}
& V^{C P L}=\min _{\substack{v \\
\text { S.t }}} v_{11}(1)+v_{21}(1) \\
& (C P L) \quad v_{11}(1)+v_{21}(1) \geq \max \left\{p_{1}\left(S_{1}\right) f_{1}, p_{2}\left(S_{2}\right) f_{2}, p_{1}\left(S_{3}\right) f_{1}+p_{2}\left(S_{3}\right) f_{2}, 0\right\} \\
& v_{11}(1)+v_{21}(0) \geq \max \left\{p_{1}\left(S_{1}\right) f_{1}, 0\right\} \\
& v_{11}(0)+v_{21}(1) \geq \max \left\{p_{2}\left(S_{2}\right) f_{2}, 0\right\} \\
& v_{11}(0)+v_{21}(0) \geq 0 \text {. }
\end{aligned}
$$

Note that the first, second, third and fourth constraints correspond to the states vectors $[1,1],[1,0]$, $[0,1]$ and $[0,0]$, respectively. It is easy to verify that an optimal solution to $(C P L)$ is $v_{11}(1)=5, v_{11}(0)=$ $10 / 11, v_{21}(1)=0, v_{21}(0)=0$ and we have $V^{C P L}=5<V^{C L R}$. Therefore, the Lagrangian relaxation approach with product and time-specific Lagrange multipliers is weaker than the piecewise-linear approximation for choice-based network RM.

\begin{tabular}{ccc}
$S$ & $p_{1}(S)$ & $p_{2}(S)$ \\
\hline$\{1\}$ & $1 / 2$ & 0 \\
$\{2\}$ & 0 & $10 / 11$ \\
$\{1,2\}$ & $1 / 12$ & $10 / 12$
\end{tabular}

TABLE 2. Choice probabilities for the example where $V^{C P L}<V^{C L R}$.

Extension to an overbooking model: In this section we sketch how the results can be extended to a network RM model with overbooking studied in Karaesmen and van Ryzin [8] and Erdelyi and Topaloglu [5]. Now, an accepted booking request shows up at the end of the sales horizon with a certain probability. The resource requirements of the show-ups can potentially exceed the available amounts, in which case we have to decide which of the show-ups are denied service. We incur a penalty cost if we deny service to a show-up and our objective is to maximize the total expected profit, which is the difference between the total expected revenues and the denied service costs. 
Let $x_{j}$ denote the number of sales of product $j$ and $\boldsymbol{x}=\left[x_{j}\right]$ be the vector of the number of sales. Let $\tilde{V}_{\tau+1}(\boldsymbol{x})$ denote the terminal revenue including the total denied service penalty cost when we have $\boldsymbol{x}$ reservations at the end of the booking period. $\tilde{V}_{\tau+1}(\boldsymbol{x})$ can be potentially obtained by solving a linear program that minimizes the total denied service cost subject to resource capacity constraints; see for example Erdelyi and Topaloglu [5]. The optimality equation is

$$
\tilde{V}_{t}(\boldsymbol{x})=\max _{\boldsymbol{u} \in\{0,1\}^{n}} \sum_{j} p_{j, t} u_{j}\left[f_{j}+\tilde{V}_{t+1}\left(\boldsymbol{x}+\boldsymbol{e}^{j}\right)-\tilde{V}_{t+1}(\boldsymbol{x})\right]+\tilde{V}_{t+1}(\boldsymbol{x}) .
$$

where $\boldsymbol{e}^{j}$ is an $n$-dimensional $0-1$ vector with a 1 at the $j$ th position and 0 elsewhere.

We make the following assumption on the terminal revenue (the denied-boarding cost): $\tilde{V}_{\tau+1}(\boldsymbol{x})=$ $\hat{V}_{\tau+1}(A \boldsymbol{x})$, where $A$ is the resource-product incidence matrix, the $(i, j)$ th element of which indicates if product $j$ consumes resource $i$. That is, the denied boarding cost is not a function of the number of reservations; it only depends on the total capacity used up on each resource. This assumption is primarily driven by tractability considerations and used also, for example, in Karaesmen and van Ryzin [8] and Erdelyi and Topaloglu [5].

Let $\boldsymbol{y}=A \boldsymbol{x}$ and note that $y_{i}$ gives the total capacity consumed on resource $i$, when the sales vector is $\boldsymbol{x}$. With the above assumption on the terminal revenue, the optimality equation can be equivalently written as

$$
\hat{V}_{t}(\boldsymbol{y})=\max _{\boldsymbol{u} \in\{0,1\}^{n}} \sum_{j} p_{j, t} u_{j}\left[f_{j}+\hat{V}_{t+1}\left(\boldsymbol{y}+\sum_{i \in \mathcal{I}_{j}} \boldsymbol{e}^{i}\right)-\hat{V}_{t+1}(\boldsymbol{y})\right]+\hat{V}_{t+1}(\boldsymbol{y})
$$

where $\boldsymbol{e}^{i}$ is an $m$-dimensional $0-1$ vector with a 1 at the $i$ th position and 0 elsewhere.

We consider the following piecewise-linear approximation: $\hat{V}_{t}(\boldsymbol{y}) \approx \sum_{i} \hat{v}_{i, t}\left(y_{i}\right)$, where we impose a restriction that $\hat{v}_{i, t}(\cdot)$ are discrete-concave functions (but not necessarily increasing). Also, let $c_{i}$ be a large enough number that the denied-boarding costs are prohibitively high (equivalently, revenue losses are huge) if the capacity consumption on resource $i$ exceeds $c_{i}$. That is, $\hat{v}_{i \tau}\left(c_{i}\right)$ is finite but $\hat{v}_{i \tau}\left(c_{i}+1\right)$ is (assumed to be) $-\infty$. Letting $\mathcal{Y}_{i}=\left\{0, \ldots, c_{i}\right\}$ and $\mathcal{Y}=\prod_{i} \mathcal{Y}_{i}$, the linear program associated with the piecewise-linear approximation is

$$
\begin{aligned}
& \hat{V}^{O P L}=\min _{\substack{\hat{v} \\
\text { s.t }}} \quad \sum_{i} \hat{v}_{i, 1}(0) \\
& (O P L) \\
& \sum_{i} \hat{v}_{i, t}\left(y_{i}\right) \geq \quad \sum_{j} p_{j, t} u_{j}\left[f_{j}+\sum_{i \in \mathcal{I}_{j}}\left\{\hat{v}_{i, t+1}\left(y_{i}+1\right)-\hat{v}_{i, t+1}\left(y_{i}\right)\right\}\right] \\
& +\sum_{i} \hat{v}_{i, t+1}\left(y_{i}\right) \quad \forall t, \boldsymbol{y} \in \mathcal{Y}, \boldsymbol{u} \in\{0,1\}^{n} \\
& \hat{v}_{i, t}\left(y_{i}\right)-\hat{v}_{i, t}\left(y_{i}-1\right) \leq \hat{v}_{i, t}\left(y_{i}-1\right)-\hat{v}_{i, t}\left(y_{i}-2\right) \quad \forall t, i, y_{i} \in \mathcal{Y}_{i} .
\end{aligned}
$$

where the objective function reflects the fact that we have no reservations and hence no capacity consumption at the start of the booking period.

Let $r_{i}=c_{i}-y_{i}$ denote the remaining capacity on resource $i$. Note that since $y_{i} \in \mathcal{Y}_{i}$, we have $r_{i} \in \mathcal{Y}_{i}$ as well. Let $v_{i, t}\left(r_{i}\right)=\hat{v}_{i, t}\left(c_{i}-r_{i}\right)=\hat{v}_{i, t}\left(y_{i}\right)$. Since $\hat{v}_{i, t}(\cdot)$ is assumed to be concave, it follows that $v_{i, t}(\cdot)$ is also concave. Therefore, $v_{i, t}\left(r_{i}\right)-v_{i, t}\left(r_{i}-1\right)$, the marginal value of capacity on resource $i$, is nonincreasing. Making the change of variables, linear program $(O P L)$ can be equivalently written as

$$
\begin{gathered}
V^{O P L}=\min _{\substack{v \\
\text { s.t }}} \quad \sum_{i} v_{i, 1}\left(c_{i}\right) \\
\quad \begin{aligned}
\sum_{i} v_{i, t}\left(r_{i}\right) \geq & \sum_{j} p_{j, t} u_{j}\left[f_{j}+\sum_{i \in \mathcal{I}_{j}}\left\{v_{i, t+1}\left(r_{i}-1\right)-v_{i, t+1}\left(r_{i}\right)\right\}\right] \\
& +\sum_{i} v_{i, t+1}\left(r_{i}\right) \quad \forall t, \boldsymbol{r} \in \mathcal{Y}, \boldsymbol{u} \in\{0,1\}^{n}
\end{aligned} \\
\\
v_{i, t}\left(r_{i}\right)-v_{i, t}\left(r_{i}-1\right) \leq v_{i, t}\left(r_{i}-1\right)-v_{i, t}\left(r_{i}-2\right) \quad \forall t, i, r_{i} \in \mathcal{Y}_{i} .
\end{gathered}
$$


The linear program $\left(O P L_{e q}\right)$ is almost similar to $(P L)$, the linear program associated the piecewiselinear approximation for the network revenue management problem where all reservations show up, except that the concavity conditions need not be redundant and the acceptance decisions are not constrained by the remaining capacities on the resources. However, notice that we still have 1) a threshold type optimal control, since $u_{j}=1$ only if $f_{j}$ exceeds $\sum_{i \in \mathcal{I}_{j}} v_{i, t+1}\left(r_{i}\right)-v_{i, t+1}\left(r_{i}-1\right)$ and 2) the marginal value of capacity, $v_{i, t+1}\left(r_{i}\right)-v_{i, t+1}\left(r_{i}-1\right)$, is nonincreasing by the concavity of $v_{i, t+1}(\cdot)$. The minimal-binding-constraints argument can be used to show that the separation problem decomposes by resource. 